\title{
SINGLE BLOW-UP SOLUTIONS FOR A SLIGHTLY SUBCRITICAL BIHARMONIC EQUATION
}

\author{
KHALIL EL MEHDI
}

Received 29 October 2004; Accepted 20 January 2005

We consider a biharmonic equation under the Navier boundary condition and with a nearly critical exponent $\left(P_{\varepsilon}\right): \Delta^{2} u=u^{9-\varepsilon}, u>0$ in $\Omega$ and $u=\Delta u=0$ on $\partial \Omega$, where $\Omega$ is a smooth bounded domain in $\mathbb{R}^{5}, \varepsilon>0$. We study the asymptotic behavior of solutions of $\left(P_{\varepsilon}\right)$ which are minimizing for the Sobolev quotient as $\varepsilon$ goes to zero. We show that such solutions concentrate around a point $x_{0} \in \Omega$ as $\varepsilon \rightarrow 0$, moreover $x_{0}$ is a critical point of the Robin's function. Conversely, we show that for any nondegenerate critical point $x_{0}$ of the Robin's function, there exist solutions of $\left(P_{\varepsilon}\right)$ concentrating around $x_{0}$ as $\varepsilon \rightarrow 0$.

Copyright (C) 2006 Khalil El Mehdi. This is an open access article distributed under the Creative Commons Attribution License, which permits unrestricted use, distribution, and reproduction in any medium, provided the original work is properly cited.

\section{Introduction and results}

Let us consider the following biharmonic equation under the Navier boundary condition

$$
\begin{aligned}
\Delta^{2} u & =u^{p-\varepsilon}, & & u>0 \text { in } \Omega \\
\Delta u & =u=0 & & \text { on } \partial \Omega,
\end{aligned}
$$

where $\Omega$ is a smooth bounded domain in $\mathbb{R}^{n}, n \geq 5, \varepsilon$ is a small positive parameter, and $p+1=2 n /(n-4)$ is the critical Sobolev exponent of the embedding $H^{2}(\Omega) \cap H_{0}^{1}(\Omega) \hookrightarrow$ $L^{2 n /(n-4)}(\Omega)$.

It is known that $\left(Q_{\varepsilon}\right)$ is related to the limiting problem $\left(Q_{0}\right)$ (when $\varepsilon=0$ ) which exhibits a lack of compactness and gives rise to solutions of $\left(Q_{\varepsilon}\right)$ which blow up as $\varepsilon \rightarrow 0$. The interest of the limiting problem $\left(Q_{0}\right)$ grew from its resemblance to some geometric equations involving Paneitz operator and which have widely been studied in these last years (for details one can see $[4,6,10,12-14,17]$ and references therein).

Several authors have studied the existence and behavior of blowing up solutions for the corresponding second order elliptic problem (see, e.g., [1, 3, 9, 18, 21, 22, 24-26] and references therein). In sharp contrast to this, very little is known for fourth order elliptic equations. In this paper we are mainly interested in the asymptotic behavior and 
the existence of solutions of $\left(Q_{\varepsilon}\right)$ which blow up around one point, and the location of this blow up point as $\varepsilon \rightarrow 0$.

The existence of solutions of $\left(Q_{\varepsilon}\right)$ for all $\varepsilon \in(0, p-1)$ is well known for any domain $\Omega$ (see, e.g., [16]). For $\varepsilon=0$, the situation is more complex, Van Der Vorst showed in [28] that if $\Omega$ is starshaped $\left(Q_{0}\right)$ has no solution whereas Ebobisse and Ould Ahmedou proved in [15] that $\left(Q_{0}\right)$ has a solution provided that some homology group of $\Omega$ is nontrivial. This topological condition is sufficient, but not necessary, as examples of contractible domains $\Omega$ on which a solution exists show [19].

In view of this qualitative change in the situation when $\varepsilon=0$, it is interesting to study the asymptotic behavior of the subcritical solution $u_{\varepsilon}$ of $\left(Q_{\varepsilon}\right)$ as $\varepsilon \rightarrow 0$. Chou and Geng [11], and Geng [20] made a first study, when $\Omega$ is strictly convex. The convexity assumption was needed in their proof in order to apply the method of moving planes (MMP for short) in proving a priori estimate near the boundary. Notice that in the Laplacian case (see [21]), the MMP has been used to show that blow up points are away from the boundary of the domain. The process is standard if domains are convex. For nonconvex regions, the MMP still works in the Laplacian case through the applications of Kelvin transformations [21]. For $\left(Q_{\varepsilon}\right)$, the MMP also works for convex domains [11]. However, for nonconvex domains, a Kelvin transformation does not work for $\left(Q_{\varepsilon}\right)$ because the Navier boundary condition is not invariant under the Kelvin transformation of biha rmonic operator. In [5], Ben Ayed and El Mehdi removed the convexity assumption of Chou and Geng for higher dimensions, that is $n \geq 6$. The aim of this paper is to prove that the results of [5] are true in dimension 5. In order to state precisely our results, we need to introduce some notations.

We consider the following problem

$$
\begin{array}{rlrl}
\Delta^{2} u & =u^{9-\varepsilon}, & & u>0 \text { in } \Omega \\
\Delta u=u=0 & & \text { on } \partial \Omega,
\end{array}
$$

where $\Omega$ is a smooth bounded domain in $\mathbb{R}^{5}$ and $\varepsilon$ is a small positive parameter.

Let us define on $\Omega$ the following Robin's function

$$
\varphi(x)=H(x, x), \quad \text { with } H(x, y)=|x-y|^{-1}-G(x, y), \text { for }(x, y) \in \Omega \times \Omega,
$$

where $G$ is the Green's function of $\Delta^{2}$, that is,

$$
\begin{aligned}
& \forall x \in \Omega \quad \Delta^{2} G(x, \cdot)=c \delta_{x} \quad \text { in } \Omega \\
& \Delta G(x, \cdot)=G(x, \cdot)=0 \quad \text { on } \partial \Omega,
\end{aligned}
$$

where $\delta_{x}$ denotes the Dirac mass at $x$ and $c=3 \omega_{5}$, with $\omega_{5}$ is the area of the unit sphere of $\mathbb{R}^{5}$. For $\lambda>0$ and $a \in \mathbb{R}^{5}$, let

$$
\delta_{a, \lambda}(x)=\frac{c_{0} \lambda^{1 / 2}}{\left(1+\lambda^{2}|x-a|^{2}\right)^{1 / 2}}, \quad c_{0}=(105)^{1 / 8} .
$$

It is well known (see [23]) that $\delta_{a, \lambda}$ are the only solutions of

$$
\Delta^{2} u=u^{9}, \quad u>0 \text { in } \mathbb{R}^{5}
$$


and are also the only minimizers of the Sobolev inequality on the whole space, that is

$$
S=\inf \left\{|\Delta u|_{L^{2}\left(\mathbb{R}^{5}\right)}^{2}|u|_{L^{10}\left(\mathbb{R}^{5}\right)}^{-2} \text {, s.t. } \Delta u \in L^{2}, u \in L^{10}, u \neq 0\right\} .
$$

We denote by $P \delta_{a, \lambda}$ the projection of $\delta_{a, \lambda}$ on $\mathcal{H}(\Omega):=H^{2}(\Omega) \cap H_{0}^{1}(\Omega)$, defined by

$$
\Delta^{2} P \delta_{a, \lambda}=\Delta^{2} \delta_{a, \lambda} \quad \text { in } \Omega, \quad \Delta P \delta_{a, \lambda}=P \delta_{a, \lambda}=0 \quad \text { on } \partial \Omega .
$$

Let

$$
\begin{gathered}
\theta_{a, \lambda}=\delta_{a, \lambda}-P \delta_{a, \lambda}, \\
\|u\|=\left(\int_{\Omega}|\Delta u|^{2}\right)^{1 / 2}, \quad\langle u, v\rangle=\int_{\Omega} \Delta u \Delta v, \quad u, v \in H^{2}(\Omega) \cap H_{0}^{1}(\Omega) \\
\|u\|_{q}=|u|_{L^{q}(\Omega)} .
\end{gathered}
$$

Thus we have the following result.

Theorem 1.1. Let $\left(u_{\varepsilon}\right)$ be a solution of $\left(P_{\varepsilon}\right)$, and assume that

$$
\left\|u_{\varepsilon}\right\|^{2}\left\|u_{\varepsilon}\right\|_{10-\varepsilon}^{-2} \longrightarrow S \text { as } \varepsilon \longrightarrow 0,
$$

where $S$ is the best Sobolev constant in $\mathbb{R}^{5}$ defined by (1.5). Then (up to a subsequence) there exist $a_{\varepsilon} \in \Omega, \lambda_{\varepsilon}>0, \alpha_{\varepsilon}>0$ and $v_{\varepsilon}$ such that $u_{\varepsilon}$ can be written as

$$
u_{\varepsilon}=\alpha_{\varepsilon} P \delta_{a_{\varepsilon}, \lambda_{\varepsilon}}+v_{\varepsilon}
$$

with $\alpha_{\varepsilon} \rightarrow 1,\left\|v_{\varepsilon}\right\| \rightarrow 0, a_{\varepsilon} \in \Omega$ and $\lambda_{\varepsilon} d\left(a_{\varepsilon}, \partial \Omega\right) \rightarrow+\infty$ as $\varepsilon \rightarrow 0$.

In addition, $a_{\varepsilon}$ converges to a critical point $x_{0} \in \Omega$ of $\varphi$ and we have

$$
\lim _{\varepsilon \rightarrow 0} \varepsilon\left\|u_{\varepsilon}\right\|_{L^{\infty}(\Omega)}^{2}=\left(c_{1} c_{0}^{2} / c_{2}\right) \varphi\left(x_{0}\right),
$$

where $c_{1}=c_{0}^{10} \int_{\mathbb{R}^{5}}\left(d x /\left(1+|x|^{2}\right)^{9 / 2}\right), c_{2}=c_{0}^{10} \int_{\mathbb{R}^{5}}\left(\log \left(1+|x|^{2}\right)\left(1-|x|^{2}\right) /\left(1+|x|^{2}\right)^{6}\right) d x$ and $c_{0}=(105)^{1 / 8}$.

Our next result provides a kind of converse to Theorem 1.1.

Theorem 1.2. Assume that $x_{0} \in \Omega$ is a nondegenerate critical point of $\varphi$. Then there exists an $\varepsilon_{0}>0$ such that for each $\varepsilon \in\left(0, \varepsilon_{0}\right],\left(P_{\varepsilon}\right)$ has a solution of the form

$$
u_{\varepsilon}=\alpha_{\varepsilon} P \delta_{a_{\varepsilon}, \lambda_{\varepsilon}}+v_{\varepsilon}
$$

with $\alpha_{\varepsilon} \rightarrow 1,\left\|v_{\varepsilon}\right\| \rightarrow 0, a_{\varepsilon} \rightarrow x_{0}$ and $\lambda_{\varepsilon} d\left(a_{\varepsilon}, \partial \Omega\right) \rightarrow+\infty$ as $\varepsilon \rightarrow 0$.

Our strategy to prove the above results is the same as in higher dimensions. However, as usual in elliptic equations involving critical Sobolev exponent, we need more refined estimates of the asymptotic profiles of solutions when $\varepsilon \rightarrow 0$ to treat the lower dimensional case. Such refined estimates, which are of self interest, are highly nontrivial and use in a crucial way careful expansions of the Euler-Lagrange functional associated to $\left(P_{\varepsilon}\right)$, and its gradient near a small neighborhood of highly concentrated functions. To perform such 
4 Single blow-up solutions for a biharmonic equation

expansions we make use of the techniques developed by Bahri [2] and Rey [25, 27] in the framework of the Theory of critical points at infinity.

The outline of the paper is the following: in Section 2 we perform some crucial estimates needed in our proofs and Section 3 is devoted to the proof of our results.

\section{Some crucial estimates}

In this section, we prove some crucial estimates which will play an important role in proving our results. We first recall some results.

Proposition 2.1 [8]. Let $a \in \Omega$ and $\lambda>0$ such that $\lambda d(a, \partial \Omega)$ is large enough. For $\theta_{(a, \lambda)}=$ $\delta_{(a, \lambda)}-P \delta_{(a, \lambda)}$, we have the following estimates

$$
0 \leq \theta_{(a, \lambda)} \leq \delta_{(a, \lambda)}, \quad \theta_{(a, \lambda)}=c_{0} \lambda^{-1 / 2} H(a, \cdot)+f_{(a, \lambda)},
$$

where $f_{(a, \lambda)}$ satisfies

$$
f_{(a, \lambda)}=O\left(\frac{1}{\lambda^{5 / 2} d^{3}}\right), \quad \lambda \frac{\partial f_{(a, \lambda)}}{\partial \lambda}=O\left(\frac{1}{\lambda^{5 / 2} d^{3}}\right), \quad \frac{1}{\lambda} \frac{\partial f_{(a, \lambda)}}{\partial a}=O\left(\frac{1}{\lambda^{7 / 2} d^{4}}\right),
$$

where $d$ is the distance $d(a, \partial \Omega)$,

$$
\begin{array}{cl}
\left|\theta_{(a, \lambda)}\right|_{L^{10}}=O\left((\lambda d)^{-1 / 2}\right), & \left\|\theta_{(a, \lambda)}\right\|=O\left((\lambda d)^{-1 / 2}\right), \\
\left|\lambda \frac{\partial \theta_{(a, \lambda)}}{\partial \lambda}\right|_{L^{10}}=O\left(\frac{1}{(\lambda d)^{1 / 2}}\right), & \left|\frac{1}{\lambda} \frac{\partial \theta_{(a, \lambda)}}{\partial a}\right|_{L^{10}}=O\left(\frac{1}{(\lambda d)^{3 / 2}}\right) .
\end{array}
$$

Proposition 2.2 [5]. Let $u_{\varepsilon}$ be a solution of $\left(P_{\varepsilon}\right)$ which satisfies $(H)$. Then, there exist $a_{\varepsilon} \in \Omega, \alpha_{\varepsilon}>0, \lambda_{\varepsilon}>0$ and $v_{\varepsilon}$ such that

$$
u_{\varepsilon}=\alpha_{\varepsilon} P \delta_{a_{\varepsilon}, \lambda_{\varepsilon}}+v_{\varepsilon}
$$

with $\alpha_{\varepsilon} \rightarrow 1, \lambda_{\varepsilon} d\left(a_{\varepsilon}, \partial \Omega\right) \rightarrow \infty, c_{0}^{-2}\left\|u_{\varepsilon}\right\|_{\infty}^{2} / \lambda_{\varepsilon} \rightarrow 1,\left\|u_{\varepsilon}\right\|_{\infty}^{\varepsilon} \rightarrow 1$ and $\left\|v_{\varepsilon}\right\| \rightarrow 0$.

Furthermore, $v_{\varepsilon} \in E_{\left(a_{\varepsilon}, \lambda_{\varepsilon}\right)}$ which is the set of $v \in \mathcal{H}(\Omega)$ such that

$$
\left\langle v, P \delta_{a_{\varepsilon}, \lambda_{\varepsilon}}\right\rangle=\left\langle v, \partial P \delta_{a_{\varepsilon}, \lambda_{\varepsilon}} / \partial \lambda_{\varepsilon}\right\rangle=0, \quad\left\langle v_{\varepsilon}, \partial P \delta_{a_{\varepsilon}, \lambda_{\varepsilon}} / \partial a\right\rangle=0 .
$$

Lemma 2.3 [5]. $\lambda_{\varepsilon}^{\varepsilon}=1+o(1)$ as $\varepsilon$ goes to zero implies that

$$
\delta_{\varepsilon}^{-\varepsilon}-c_{0}^{-\varepsilon} \lambda_{\varepsilon}^{\varepsilon(4-n) / 2}=O\left(\varepsilon \log \left(1+\lambda_{\varepsilon}^{2}\left|x-a_{\varepsilon}\right|^{2}\right)\right) \quad \text { in } \Omega,
$$

where $\delta_{\varepsilon}=\delta_{a_{\varepsilon}, \lambda_{\varepsilon}}$ and $d_{\varepsilon}=d\left(a_{\varepsilon}, \partial \Omega\right)$.

Proposition 2.4 [5]. Let $\left(u_{\varepsilon}\right)$ be a solution of $\left(P_{\varepsilon}\right)$ which satisfies $(H)$. Then $v_{\varepsilon}$ occurring in Proposition 2.2 satisfies

$$
\left\|v_{\varepsilon}\right\| \leq C\left(\varepsilon+\left(\lambda_{\varepsilon} d_{\varepsilon}\right)^{-1}\right),
$$

where $C$ is a positive constant independent of $\varepsilon$. 
Now, we are going to state and prove the crucial estimates needed in the proof of our theorems. In order to simplify the notations, we set $\delta_{\varepsilon}=\delta_{a_{\varepsilon}, \lambda_{\varepsilon}}, P \delta_{\varepsilon}=P \delta_{a_{\varepsilon}, \lambda_{\varepsilon}}, \theta_{\varepsilon}=\theta_{a_{\varepsilon}, \lambda_{\varepsilon}}$ and $d_{\varepsilon}=d\left(a_{\varepsilon}, \partial \Omega\right)$.

LEMMA 2.5. For $\varepsilon$ small, we have the following estimates

(i) $\int_{\Omega} \delta_{\varepsilon}^{9}\left(1 / \lambda_{\varepsilon}\right)\left(\partial P \delta_{\varepsilon} / \partial a\right)=-\left(c_{1} / 2 \lambda_{\varepsilon}^{2}\right)(\partial H / \partial a)\left(a_{\varepsilon}, a_{\varepsilon}\right)+O\left(1 /\left(\lambda_{\varepsilon} d_{\varepsilon}\right)^{3}\right)$,

(ii) $\int_{\Omega} P \delta_{\varepsilon}^{9-\varepsilon}\left(1 / \lambda_{\varepsilon}\right)\left(\partial P \delta_{\varepsilon} / \partial a\right)=-\left(c_{1} / \lambda_{\varepsilon}^{2+\varepsilon / 2}\right)(\partial H / \partial a)\left(a_{\varepsilon}, a_{\varepsilon}\right)+O\left(1 /\left(\lambda_{\varepsilon} d_{\varepsilon}\right)^{3}+\varepsilon /\left(\lambda_{\varepsilon} d_{\varepsilon}\right)^{2}\right)$, where $c_{1}$ is the constant defined in Theorem 1.1.

Proof. Notice that

$$
\int_{\Omega \backslash B_{\varepsilon}} \delta_{\varepsilon}^{10}=O\left(\frac{1}{\left(\lambda_{\varepsilon} d_{\varepsilon}\right)^{5}}\right)
$$

Thus, we have, for $1 \leq k \leq 5$

$$
\int_{\Omega} \delta_{\varepsilon}^{9} \frac{1}{\lambda_{\varepsilon}} \frac{\partial P \delta_{\varepsilon}}{\partial a_{k}}=\int_{\Omega} \delta_{\varepsilon}^{9} \frac{1}{\lambda_{\varepsilon}} \frac{\partial \delta_{\varepsilon}}{\partial a_{k}}-\int_{\Omega} \delta_{\varepsilon}^{9} \frac{1}{\lambda_{\varepsilon}} \frac{\partial \theta_{\varepsilon}}{\partial a_{k}}=-\int_{B_{\varepsilon}} \delta_{\varepsilon}^{9} \frac{1}{\lambda_{\varepsilon}} \frac{\partial \theta_{\varepsilon}}{\partial a_{k}}+O\left(\frac{1}{\left(\lambda_{\varepsilon} d_{\varepsilon}\right)^{5}}\right),
$$

where $B_{\varepsilon}=B\left(a_{\varepsilon}, d_{\varepsilon}\right)$. Expanding $\partial \theta_{\varepsilon} / \partial a_{k}$ around $a_{\varepsilon}$ and using Proposition 2.1, we obtain

$$
\int_{B_{\varepsilon}} \delta_{\varepsilon}^{9} \frac{1}{\lambda_{\varepsilon}} \frac{\partial \theta_{\varepsilon}}{\partial a_{k}}=\frac{c_{0}}{2 \lambda_{\varepsilon}^{3 / 2}} \frac{\partial H\left(a_{\varepsilon}, a_{\varepsilon}\right)}{\partial a} \int_{B_{\varepsilon}} \delta_{\varepsilon}^{9}+O\left(\frac{1}{\left(\lambda_{\varepsilon} d_{\varepsilon}\right)^{3}}\right)
$$

Estimating the integral on the right-hand side in (2.9) and using (2.8), we easily derive claim (i). To prove claim (ii), we write

$$
\begin{aligned}
\int_{\Omega} P \delta_{\varepsilon}^{9-\varepsilon} \frac{1}{\lambda_{\varepsilon}} \frac{\partial P \delta_{\varepsilon}}{\partial a_{k}}= & \int_{\Omega} \delta_{\varepsilon}^{9-\varepsilon} \frac{1}{\lambda_{\varepsilon}} \frac{\partial \delta_{\varepsilon}}{\partial a_{k}}-\int_{\Omega} \delta_{\varepsilon}^{9-\varepsilon} \frac{1}{\lambda_{\varepsilon}} \frac{\partial \theta_{\varepsilon}}{\partial a_{k}}-(9-\varepsilon) \int_{\Omega} \delta_{\varepsilon}^{8-\varepsilon} \theta_{\varepsilon} \frac{1}{\lambda} \frac{\partial \delta_{\varepsilon}}{\partial a_{k}} \\
& +\frac{(9-\varepsilon)(8-\varepsilon)}{2} \int_{\Omega} \delta_{\varepsilon}^{7-\varepsilon} \theta_{\varepsilon}^{2} \frac{1}{\lambda} \frac{\partial \delta_{\varepsilon}}{\partial a_{k}}+O\left(\int_{\Omega} \delta_{\varepsilon}^{8-\varepsilon} \theta_{\varepsilon}\left|\frac{1}{\lambda_{\varepsilon}} \frac{\partial \theta_{\varepsilon}}{\partial a_{k}}\right|+\int \delta_{\varepsilon}^{7-\varepsilon} \theta_{\varepsilon}^{3}\right)
\end{aligned}
$$

and we have to estimate each term on the right-hand side of (2.10).

Using Proposition 2.1 and Lemma 2.3, we have

$$
\begin{gathered}
\int_{\Omega} \delta_{\varepsilon}^{7-\varepsilon} \theta_{\varepsilon}^{3} \leq c\left\|\theta_{\varepsilon}\right\|_{\infty}^{3} \int \delta_{\varepsilon}^{7}=O\left(\frac{1}{\left(\lambda_{\varepsilon} d_{\varepsilon}\right)^{3}}\right), \\
\int_{\Omega} \delta_{\varepsilon}^{8-\varepsilon} \theta_{\varepsilon}\left|\frac{1}{\lambda_{\varepsilon}} \frac{\partial \theta_{\varepsilon}}{\partial a_{k}}\right| \leq c|| \theta_{\varepsilon} \|_{\infty}|| \frac{1}{\lambda_{\varepsilon}} \frac{\partial \theta_{\varepsilon}}{\partial a_{k}}||_{\infty} \int_{\Omega} \delta_{\varepsilon}^{8}=O\left(\frac{1}{\left(\lambda_{\varepsilon} d_{\varepsilon}\right)^{3}}\right) .
\end{gathered}
$$

We also have

$$
\int_{\Omega} \delta_{\varepsilon}^{9-\varepsilon} \frac{1}{\lambda_{\varepsilon}} \frac{\partial \delta_{\varepsilon}}{\partial a_{k}}=\int_{\Omega \backslash B_{\varepsilon}} \delta_{\varepsilon}^{9-\varepsilon} \frac{1}{\lambda_{\varepsilon}} \frac{\partial \delta_{\varepsilon}}{\partial a_{k}}=O\left(\frac{1}{\left(\lambda_{\varepsilon} d_{\varepsilon}\right)^{5}}\right)
$$


6 Single blow-up solutions for a biharmonic equation

Expanding $\theta_{\varepsilon}$ around $a_{\varepsilon}$ and using Proposition 2.1 and Lemma 2.3, we obtain

$$
\begin{gathered}
9 \int_{B_{\varepsilon}} \delta_{\varepsilon}^{8-\varepsilon} \theta_{\varepsilon} \frac{1}{\lambda_{\varepsilon}} \frac{\partial \delta_{\varepsilon}}{\partial a_{k}}=\frac{c_{1}}{2 \lambda_{\varepsilon}^{2+\varepsilon / 2}} \frac{\partial H\left(a_{\varepsilon}, a_{\varepsilon}\right)}{\partial a}+O\left(\frac{1}{\left(\lambda_{\varepsilon} d_{\varepsilon}\right)^{3}}+\frac{\varepsilon}{\left(\lambda_{\varepsilon} d_{\varepsilon}\right)^{2}}\right), \\
\int_{B_{\varepsilon}} \delta_{\varepsilon}^{7-\varepsilon} \theta_{\varepsilon}^{2} \frac{1}{\lambda_{\varepsilon}} \frac{\partial \delta_{\varepsilon}}{\partial a_{k}}=O\left(\frac{1}{\left(\lambda_{\varepsilon} d_{\varepsilon}\right)^{3}}\right) .
\end{gathered}
$$

In the same way, we find

$$
\int_{\Omega} \delta_{\varepsilon}^{9-\varepsilon} \frac{1}{\lambda_{\varepsilon}} \frac{\partial \theta_{\varepsilon}}{\partial a_{k}}=\frac{c_{1}}{2 \lambda_{\varepsilon}^{2+\varepsilon / 2}} \frac{\partial H\left(a_{\varepsilon}, a_{\varepsilon}\right)}{\partial a}+O\left(\frac{1}{\left(\lambda_{\varepsilon} d_{\varepsilon}\right)^{3}}+\frac{\varepsilon}{\left(\lambda_{\varepsilon} d_{\varepsilon}\right)^{2}}\right) .
$$

Combining (2.10)-(2.14), we obtain claim (ii).

To improve the estimates of the integrals involving $v_{\varepsilon}$, we use an idea of Rey [27], namely we write

$$
v_{\varepsilon}=\Pi v_{\varepsilon}+w_{\varepsilon}
$$

where $\Pi v_{\varepsilon}$ denotes the projection of $v_{\varepsilon}$ onto $H^{2} \cap H_{0}^{1}\left(B_{\varepsilon}\right)$, that is

$$
\Delta^{2} \Pi v_{\varepsilon}=\Delta^{2} v_{\varepsilon} \quad \text { in } B_{\varepsilon} ; \quad \Delta \Pi v_{\varepsilon}=\Pi v_{\varepsilon}=0 \quad \text { on } \partial B_{\varepsilon}
$$

where $B_{\varepsilon}=B\left(a_{\varepsilon}, d_{\varepsilon}\right)$. We split $\Pi v_{\varepsilon}$ in an even part $\Pi v_{\varepsilon}^{e}$ and an odd part $\Pi v_{\varepsilon}^{o}$ with respect to $\left(x-a_{\varepsilon}\right)_{k}$, thus we have

$$
v_{\varepsilon}=\Pi v_{\varepsilon}^{e}+\Pi v_{\varepsilon}^{o}+w_{\varepsilon} \quad \text { in } B_{\varepsilon} \text { with } \Delta^{2} w_{\varepsilon}=0 \text { in } B_{\varepsilon} .
$$

Notice that it is difficult to improve the estimate (2.6) of the $v_{\varepsilon}$-part of solutions. However, it is sufficient to improve the integrals involving the odd part of $v_{\varepsilon}$ with respect to $(x-$ $\left.a_{\varepsilon}\right)_{k}$, for $1 \leq k \leq 5$ and to know the exact contribution of the integrals containing the $w_{\mathcal{\varepsilon}}$-part of $v_{\varepsilon}$. Let us start by the terms involving $w_{\varepsilon}$.

Lemma 2.6. For $\varepsilon$ small, we have that

$$
\int_{B_{\varepsilon}} \delta_{\varepsilon}^{8}\left(\delta_{\varepsilon}^{-\varepsilon}-\frac{1}{c_{0}^{\varepsilon} \lambda_{\varepsilon}^{\varepsilon / 2}}\right) \frac{1}{\lambda_{\varepsilon}} \frac{\partial \delta_{\varepsilon}}{\partial a_{k}} w_{\varepsilon}=O\left(\frac{\varepsilon\left\|v_{\varepsilon}\right\|}{\left(\lambda_{\varepsilon} d_{\varepsilon}\right)^{1 / 2}}\right)
$$

Proof. Let $\psi$ be the solution of

$$
\Delta^{2} \psi=\delta_{\varepsilon}^{8}\left(\delta_{\varepsilon}^{-\varepsilon}-\frac{1}{c_{0}^{\varepsilon} \lambda_{\varepsilon}^{\varepsilon / 2}}\right) \frac{1}{\lambda_{\varepsilon}} \frac{\partial \delta_{\varepsilon}}{\partial a_{k}} \quad \text { in } B_{\varepsilon} ; \quad \Delta \psi=\psi=0 \quad \text { on } \partial B_{\varepsilon} .
$$

Thus we have

$$
I_{\varepsilon}:=\int_{B_{\varepsilon}} \Delta^{2} \psi w_{\varepsilon}=\int_{\partial B_{\varepsilon}} \frac{\partial \psi}{\partial \nu} \Delta w_{\varepsilon}+\int_{\partial B_{\varepsilon}} \frac{\partial \Delta \psi}{\partial \nu} w_{\varepsilon} .
$$


Let $G_{\varepsilon}$ be the Green's function for the biharmonic operator on $B_{\varepsilon}$ with the Navier boundary conditions, that is,

$$
\Delta^{2} G_{\varepsilon}(x, \cdot)=c \delta_{x} \quad \text { in } B_{\varepsilon} ; \quad \Delta G_{\varepsilon}(x, \cdot)=G(x, \cdot)=0 \quad \text { on } \partial B_{\varepsilon},
$$

where $c=3 w_{5}$. Therefore $\psi$ is given by

$$
\psi(y)=\int_{B_{\varepsilon}} G_{\varepsilon}(x, y) \delta_{\varepsilon}^{8}\left(\delta_{\varepsilon}^{-\varepsilon}-\frac{1}{c_{0}^{\varepsilon} \lambda_{\varepsilon}^{\varepsilon / 2}}\right) \frac{1}{\lambda_{\varepsilon}} \frac{\partial \delta_{\varepsilon}}{\partial a_{k}}, \quad y \in B_{\varepsilon}
$$

and its normal derivative by

$$
\frac{\partial \psi}{\partial \nu}(y)=\int_{B_{\varepsilon}} \frac{\partial G_{\varepsilon}}{\partial \nu}(x, y) \delta_{\varepsilon}^{8}\left(\delta_{\varepsilon}^{-\varepsilon}-\frac{1}{c_{0}^{\varepsilon} \lambda_{\varepsilon}^{\varepsilon / 2}}\right) \frac{1}{\lambda_{\varepsilon}} \frac{\partial \delta_{\varepsilon}}{\partial a_{k}}, \quad y \in \partial B_{\varepsilon} .
$$

Notice that for $y \in \partial B_{\varepsilon}$ we have the following estimates: for $x \in B_{\varepsilon} \backslash B\left(y, d_{\varepsilon} / 2\right)$, we have

$$
\frac{\partial G_{\varepsilon}}{\partial \nu}(x, y)=O\left(\frac{1}{d_{\varepsilon}^{2}}\right) ; \quad \frac{\partial \Delta G_{\varepsilon}}{\partial \nu}(x, y)=O\left(\frac{1}{d_{\varepsilon}^{4}}\right)
$$

for $x \in B_{\varepsilon} \cap B\left(y, d_{\varepsilon} / 2\right)$, we have

$$
\left|\frac{\partial G_{\varepsilon}}{\partial \nu}(x, y)\right| \leq \frac{c}{|x-y|^{2}} ; \quad\left|\frac{\partial \Delta G_{\varepsilon}}{\partial \nu}(x, y)\right| \leq \frac{c}{|x-y|^{4}}
$$

for $x \in B_{\varepsilon} \cap B\left(y, d_{\varepsilon} / 2\right)$, we have

$$
\delta_{\varepsilon}^{8}\left(\delta_{\varepsilon}^{-\varepsilon}-\frac{1}{c_{0}^{\varepsilon} \lambda_{\varepsilon}^{\varepsilon / 2}}\right) \frac{1}{\lambda_{\varepsilon}} \frac{\partial \delta_{\varepsilon}}{\partial a_{k}}=O\left(\frac{\varepsilon \log \lambda_{\varepsilon} d_{\varepsilon}}{\left(\lambda_{\varepsilon} d_{\varepsilon}\right)^{9}}\right),
$$

for $x \in B_{\varepsilon} \backslash B\left(y, d_{\varepsilon} / 2\right)$, we have

$$
\delta_{\varepsilon}^{8}\left(\delta_{\varepsilon}^{-\varepsilon}-\frac{1}{c_{0}^{\varepsilon} \lambda_{\varepsilon}^{\varepsilon / 2}}\right) \frac{1}{\lambda_{\varepsilon}} \frac{\partial \delta_{\varepsilon}}{\partial a_{k}}=O\left(\delta_{\varepsilon}^{9} \varepsilon \log \left(1+\lambda_{\varepsilon}^{2}\left|x-a_{\varepsilon}\right|^{2}\right)\right) .
$$

Therefore

$$
\left|\frac{\partial \psi}{\partial \nu}(y)\right|=O\left(\frac{\varepsilon}{\lambda_{\varepsilon}^{1 / 2} d_{\varepsilon}^{2}}\right)
$$

In the same way, we have

$$
\left|\frac{\partial \Delta \psi}{\partial \nu}(y)\right|=O\left(\frac{\varepsilon}{\lambda_{\varepsilon}^{1 / 2} d_{\varepsilon}^{4}}\right) .
$$

Using (2.20), (2.28), (2.29), we obtain

$$
I_{\varepsilon}=O\left(\frac{\varepsilon}{\lambda_{\varepsilon}^{1 / 2} d_{\varepsilon}^{2}} \int_{\partial B_{\varepsilon}}\left|\Delta w_{\varepsilon}\right|+\frac{\varepsilon}{\lambda_{\varepsilon}^{1 / 2} d_{\varepsilon}^{4}} \int_{\partial B_{\varepsilon}}\left|w_{\varepsilon}\right|\right) .
$$


8 Single blow-up solutions for a biharmonic equation

To estimate the right-hand side of (2.30), we introduce the following function

$$
\bar{w}(X)=d_{\varepsilon}^{1 / 2} w_{\varepsilon}\left(a_{\varepsilon}+d_{\varepsilon} X\right), \quad \bar{v}(X)=d_{\varepsilon}^{1 / 2} v_{\varepsilon}\left(a_{\varepsilon}+d_{\varepsilon} X\right) \quad \text { for } X \in B(0,1) .
$$

$\bar{w}$ satisfies

$$
\Delta^{2} \bar{w}=0 \quad \text { in } B:=B(0,1) ; \quad \Delta \bar{w}=\Delta \bar{v}, \quad \bar{w}=\bar{v} \text { on } \partial B
$$

We deduce that

$$
\int_{\partial B}|\Delta \bar{w}|+\int_{\partial B}|\Delta \bar{w}| \leq C\left(\int_{B}|\Delta \bar{v}|^{2}\right)^{1 / 2}=C\left(\int_{B_{\varepsilon}}\left|\Delta v_{\varepsilon}\right|^{2}\right)^{1 / 2} .
$$

But, we have

$$
\int_{\partial B}|\Delta \bar{w}|+\int_{\partial B}|\Delta \bar{w}|=\left(\frac{1}{d_{\varepsilon}}\right)^{3 / 2} \int_{\partial B_{\varepsilon}}\left|\Delta w_{\varepsilon}\right|+\left(\frac{1}{d_{\varepsilon}}\right)^{7 / 2} \int_{\partial B_{\varepsilon}}\left|w_{\varepsilon}\right| .
$$

Using (2.30), (2.33) and (2.34), the lemma follows.

LEMMa 2.7. For $\varepsilon$ small, we have

(i) $\int_{B_{\varepsilon}} \Delta\left(\left(1 / \lambda_{\varepsilon}\right)\left(\partial \Pi \delta_{\varepsilon} / \partial a_{k}\right)\right) \Delta w_{\varepsilon}=O\left(\left\|v_{\varepsilon}\right\| /\left(\lambda_{\varepsilon} d_{\varepsilon}\right)^{3 / 2}\right)$,

(ii) $\int_{B_{\varepsilon}} \delta_{\varepsilon}^{8-\varepsilon} \Pi v_{\varepsilon}^{o} w_{\varepsilon}=O\left(\left\|v_{\varepsilon}\right\|\left\|\Pi v_{\varepsilon}^{o}\right\| /\left(\lambda_{\varepsilon} d_{\varepsilon}\right)^{1 / 2}\right)$.

Proof. Using (2.17), we obtain

$$
\int_{B_{\varepsilon}} \Delta\left(\frac{1}{\lambda_{\varepsilon}} \frac{\partial \Pi \delta_{\varepsilon}}{\partial a_{k}}\right) \Delta w_{\varepsilon}=\int_{\partial B_{\varepsilon}} \frac{\partial \psi_{k}}{\partial \nu} \Delta w_{\varepsilon}, \quad \text { with } \psi_{k}=\frac{1}{\lambda_{\varepsilon}} \frac{\partial \Pi \delta_{\varepsilon}}{\partial a_{k}} .
$$

Using an integral representation for $\psi_{k}$ as in (2.23), we obtain for $y \in \partial B_{\varepsilon}$,

$$
\frac{\partial \psi}{\partial \nu}(y)=\int_{B_{\varepsilon}} \frac{\partial G_{\varepsilon}}{\partial \nu}(x, y) \Delta^{2} \psi_{k}
$$

where $G_{\varepsilon}$ is the Green's function defined in (2.21). Clearly, we have

$$
\Pi \delta_{\varepsilon}(x)=\delta_{\varepsilon}(x)-\frac{c_{0} \lambda_{\varepsilon}^{1 / 2}}{\left(1+\lambda_{\varepsilon}^{2} d_{\varepsilon}^{2}\right)^{1 / 2}}-\frac{c_{\varepsilon}\left(a_{\varepsilon}, d_{\varepsilon}\right)}{10}\left(\left|x-a_{\varepsilon}\right|^{2}-d_{\varepsilon}^{2}\right),
$$

with $c_{\varepsilon}\left(a_{\varepsilon}, d_{\varepsilon}\right)=\Delta \delta_{\varepsilon_{\mid \partial B_{\varepsilon}}}$. Thus we deduce that

$$
\frac{\partial \psi}{\partial \nu}(y)=9 \int_{B_{\varepsilon}} \frac{\partial G_{\varepsilon}}{\partial \nu}(x, y) \delta_{\varepsilon}^{8} \frac{1}{\lambda_{\varepsilon}} \frac{\partial \delta_{\varepsilon}}{\partial a_{k}} .
$$

In $B_{\varepsilon} \backslash B\left(a_{\varepsilon}, d_{\varepsilon} / 2\right)$, we argue as in (2.28) and (2.25), we obtain

$$
\int_{B_{\varepsilon}} \frac{\partial G_{\varepsilon}}{\partial \nu}(x, y) \delta_{\varepsilon}^{8} \frac{1}{\lambda_{\varepsilon}} \frac{\partial \delta_{\varepsilon}}{\partial a_{k}}=O\left(\frac{1}{\lambda_{\varepsilon}^{9 / 2} d_{\varepsilon}^{6}}\right)
$$


Furthermore, since

$$
\left|\nabla \frac{\partial G_{\varepsilon}}{\partial \nu}(x, y)\right|=O\left(\frac{1}{d_{\varepsilon}^{3}}\right) \quad \text { for }(x, y) \in B\left(a_{\varepsilon}, d_{\varepsilon} / 2\right) \times \partial B_{\varepsilon}
$$

we obtain

$$
\left|\int_{B\left(a_{\varepsilon}, d_{\varepsilon} / 2\right)} \frac{\partial G_{\varepsilon}}{\partial \nu}(x, y) \delta_{\varepsilon}^{8} \frac{1}{\lambda_{\varepsilon}} \frac{\partial \delta_{\varepsilon}}{\partial a_{k}}\right| \leq \frac{c}{d_{\varepsilon}^{3}} \int_{B\left(a_{\varepsilon}, d_{\varepsilon} / 2\right)} \delta_{\varepsilon}^{9}\left|x-a_{\varepsilon}\right|=O\left(\frac{1}{\lambda_{\varepsilon}^{3 / 2} d_{\varepsilon}^{3}}\right),
$$

where we have used the evenness of $\delta_{\varepsilon}$ and the oddness of its derivative. Thus

$$
\frac{\partial \psi_{k}}{\partial \nu}(y)=O\left(\frac{1}{\lambda_{\varepsilon}^{3 / 2} d_{\varepsilon}^{3}}\right)
$$

Using (2.35) and (2.42), we obtain

$$
\int_{B_{\varepsilon}} \Delta\left(\frac{1}{\lambda_{\varepsilon}} \frac{\partial \Pi \delta_{\varepsilon}}{\partial a_{k}}\right) \Delta w_{\varepsilon} \leq \frac{c}{\lambda_{\varepsilon}^{3 / 2} d_{\varepsilon}^{3}} \int_{\partial B_{\varepsilon}}\left|\Delta w_{\varepsilon}\right|
$$

Arguing as in (2.34), claim (i) follows. To prove claim (ii), let $\psi$ be such that

$$
\Delta^{2} \psi=\delta_{\varepsilon}^{8-\varepsilon} \Pi v_{\varepsilon}^{o} \quad \text { in } B_{\varepsilon} ; \quad \Delta \psi=\psi=0 \quad \text { on } \partial B_{\varepsilon} .
$$

We have

$$
\int_{B_{\varepsilon}} \delta_{\varepsilon}^{8-\varepsilon} \Pi v_{\varepsilon}^{o} w_{\varepsilon}=\int_{\partial B_{\varepsilon}} \frac{\partial \Delta \psi}{\partial \nu} w_{\varepsilon}+\int_{\partial B_{\varepsilon}} \frac{\partial \psi}{\partial \nu} \Delta w_{\varepsilon}
$$

As before, we prove that, for $y \in \partial B_{\varepsilon}$

$$
\frac{\partial \psi}{\partial \nu}(y)=O\left(\frac{\left\|\Pi v_{\varepsilon}^{o}\right\|}{\lambda_{\varepsilon}^{1 / 2} d_{\varepsilon}^{2}}\right), \quad \frac{\partial \Delta \psi}{\partial \nu}(y)=O\left(\frac{\left\|\Pi v_{\varepsilon}^{o}\right\|}{\lambda_{\varepsilon}^{1 / 2} d_{\varepsilon}^{4}}\right)
$$

Therefore

$$
\int_{B_{\varepsilon}} \delta_{\varepsilon}^{8-\varepsilon} \Pi v_{\varepsilon}^{o} w_{\varepsilon} \leq \frac{c\left\|\Pi v_{\varepsilon}^{o}\right\|}{\lambda_{\varepsilon}^{1 / 2} d_{\varepsilon}^{4}}\left(\frac{1}{\delta_{\varepsilon}^{3 / 2}} \int_{\partial B_{\varepsilon}}\left|w_{\varepsilon}\right|+\frac{1}{\delta_{\varepsilon}^{7 / 2}} \int_{\partial B_{\varepsilon}}\left|\Delta w_{\varepsilon}\right|\right) \leq \frac{c\left\|v_{\varepsilon}\right\|\left\|\Pi v_{\varepsilon}^{o}\right\|}{\left(\lambda_{\varepsilon} d_{\varepsilon}\right)^{1 / 2}}
$$

The proof of the lemma is completed.

LEMMA 2.8. For $\varepsilon$ small, we have

(i) $\int_{B_{\varepsilon}} \delta_{\varepsilon}^{7-\varepsilon} v_{\varepsilon}\left(1 / \lambda_{\varepsilon}\right)\left(\partial \delta_{\varepsilon} / \partial a_{k}\right)=O\left(\left\|\Pi v_{\varepsilon}^{o}\right\| / \lambda_{\varepsilon}^{1 / 2}+\left\|v_{\varepsilon}\right\| / \lambda_{\varepsilon} d_{\varepsilon}^{1 / 2}\right)$,

(ii) $\int_{B_{\varepsilon}} \delta_{\varepsilon}^{7-\varepsilon} \theta_{\varepsilon} v_{\varepsilon}\left(1 / \lambda_{\varepsilon}\right)\left(\partial \delta_{\varepsilon} / \partial a_{k}\right)=O\left(\left\|\Pi v_{\varepsilon}^{o}\right\| / \lambda_{\varepsilon} d_{\varepsilon}+\left\|v_{\varepsilon}\right\| /\left(\lambda_{\varepsilon} d_{\varepsilon}\right)^{3 / 2}\right)$.

Proof. Claim (i) can be proved in the same way as Lemma 2.6, so we omit its proof. Claim (ii) follows from Proposition 2.1 and claim (i). 
10 Single blow-up solutions for a biharmonic equation

Let us now compute the contribution of the following integral which involves $v_{\varepsilon}^{2}$.

Lemma 2.9. For $\varepsilon$ small, we have

$$
\int_{B_{\varepsilon}} \delta_{\varepsilon}^{7-\varepsilon} v_{\varepsilon}^{2} \frac{1}{\lambda_{\varepsilon}} \frac{\partial \delta_{\varepsilon}}{\partial a_{k}}=O\left(\left\|\Pi v_{\varepsilon}^{o}\right\|\left\|v_{\varepsilon}\right\|+\frac{\left\|v_{\varepsilon}\right\|^{2}}{\left(\lambda_{\varepsilon} d_{\varepsilon}\right)^{1 / 2}}\right)
$$

Proof. Using (2.17) and the fact that the even part of $v_{\varepsilon}^{2}$ has no contribution to the integrals, we obtain

$$
\int_{B_{\varepsilon}} \delta_{\varepsilon}^{7-\varepsilon} v_{\varepsilon}^{2} \frac{1}{\lambda_{\varepsilon}} \frac{\partial \delta_{\varepsilon}}{\partial a_{k}}=\int_{B_{\varepsilon}} \delta_{\varepsilon}^{7-\varepsilon} \frac{1}{\lambda_{\varepsilon}} \frac{\partial \delta_{\varepsilon}}{\partial a_{k}}\left(2 v_{\varepsilon}-w_{\varepsilon}\right) w_{\varepsilon}+O\left(\left\|\Pi v_{\varepsilon}^{o}\right\|\left\|v_{\varepsilon}\right\|\right) .
$$

Let $\Psi$ be the solution of

$$
\Delta^{2} \Psi=\delta_{\varepsilon}^{7-\varepsilon} \frac{1}{\lambda_{\varepsilon}} \frac{\partial \delta_{\varepsilon}}{\partial a_{k}}\left(2 v_{\varepsilon}-w_{\varepsilon}\right) \quad \text { in } B_{\varepsilon} ; \quad \Delta \Psi=\Psi=0 \quad \text { on } \partial B_{\varepsilon} .
$$

Thus, as in the proof of Lemma 2.6, we obtain for $y \in \partial B_{\varepsilon}$

$$
\frac{\partial \Psi}{\partial \nu}(y)=O\left(\frac{\left\|v_{\varepsilon}\right\|}{\lambda_{\varepsilon}^{1 / 2} d_{\varepsilon}^{2}}\right), \quad \frac{\partial \Delta \Psi}{\partial \nu}(y)=O\left(\frac{\left\|v_{\varepsilon}\right\|}{\lambda_{\varepsilon}^{1 / 2} d_{\varepsilon}^{4}}\right)
$$

and therefore

$$
\int_{B_{\varepsilon}} \delta_{\varepsilon}^{7-\varepsilon} \frac{1}{\lambda_{\varepsilon}} \frac{\partial \delta_{\varepsilon}}{\partial a_{k}}\left(2 v_{\varepsilon}-w_{\varepsilon}\right) w_{\varepsilon}=O\left(\frac{\left\|v_{\varepsilon}\right\|^{2}}{\left(\lambda_{\varepsilon} d_{\varepsilon}\right)^{1 / 2}}\right)
$$

Thus our lemma follows.

Next we are going to estimate the integrals involving the odd part of $v_{\varepsilon}$ with respect to $\left(x-a_{\varepsilon}\right)_{k}$, for $1 \leq k \leq 5$.

Lemma 2.10. For $\varepsilon$ small, we have

$$
\int_{B_{\varepsilon}} u_{\varepsilon}^{9-\varepsilon} \Pi v_{\varepsilon}^{o}=9 \int_{B_{\varepsilon}} \delta_{\varepsilon}^{8}\left(\Pi v_{\varepsilon}^{o}\right)^{2}+o\left(\left\|\Pi v_{\varepsilon}^{o}\right\|^{2}\right)+O\left(\left\|\Pi v_{\varepsilon}^{o}\right\|\left(\varepsilon^{3 / 2}+\frac{1}{\left(\lambda_{\varepsilon} d_{\varepsilon}\right)^{3 / 2}}\right)\right)
$$

Proof. We have

$$
\begin{aligned}
\int_{B_{\varepsilon}} u_{\varepsilon}^{9-\varepsilon} \Pi v_{\varepsilon}^{o}= & \alpha_{\varepsilon}^{9-\varepsilon} \int_{B_{\varepsilon}} P \delta_{\varepsilon}^{9-\varepsilon} \Pi v_{\varepsilon}^{o}+(9-\varepsilon) \alpha_{\varepsilon}^{8-\varepsilon} \int_{B_{\varepsilon}} P \delta_{\varepsilon}^{8-\varepsilon} v_{\varepsilon} \Pi v_{\varepsilon}^{o} \\
& +O\left(\int_{B_{\varepsilon}} P \delta_{\varepsilon}^{7-\varepsilon}\left|v_{\varepsilon}\right|^{2}\left|\Pi v_{\varepsilon}^{o}\right|+\int_{B_{\varepsilon}}\left|v_{\varepsilon}\right|^{9-\varepsilon}\left|\Pi v_{\varepsilon}^{o}\right|\right) \\
= & \alpha_{\varepsilon}^{9-\varepsilon} \int_{B_{\varepsilon}} P \delta_{\varepsilon}^{9-\varepsilon} \Pi v_{\varepsilon}^{o}+(9-\varepsilon) \alpha_{\varepsilon}^{8-\varepsilon} \int_{B_{\varepsilon}} P \delta_{\varepsilon}^{8-\varepsilon} v_{\varepsilon} \Pi v_{\varepsilon}^{o}+O\left(\left\|v_{\varepsilon}\right\|^{2}\left\|\Pi v_{\varepsilon}^{o}\right\|\right) .
\end{aligned}
$$


We estimate the two integrals on the right-hand side in (2.54). First, using Proposition 2.1 and the Holder inequality, we have

$$
\int_{B_{\varepsilon}} P \delta_{\varepsilon}^{8-\varepsilon} v_{\varepsilon} \Pi v_{\varepsilon}^{o}=\int_{B_{\varepsilon}} \delta_{\varepsilon}^{8-\varepsilon} v_{\varepsilon} \Pi v_{\varepsilon}^{o}+O\left(\frac{\left\|v_{\varepsilon}\right\|\left\|\Pi v_{\varepsilon}^{o}\right\|}{\lambda_{\varepsilon} d_{\varepsilon}}\right)=\int_{B_{\varepsilon}} \delta_{\varepsilon}^{8-\varepsilon}\left(\Pi v_{\varepsilon}^{o}\right)^{2}+\int_{B_{\varepsilon}} \delta_{\varepsilon}^{8-\varepsilon} \Pi v_{\varepsilon}^{o} w_{\varepsilon}
$$

where we have used in the last equality the evenness of $\delta_{\varepsilon}$ and $\Pi v_{\varepsilon}^{e}$ and the oddness of $\Pi v_{\varepsilon}^{o}$. By Lemmas 2.3 and 2.7 we obtain

$$
\int_{B_{\varepsilon}} P \delta_{\varepsilon}^{8-\varepsilon} v_{\varepsilon} \Pi v_{\varepsilon}^{o}=\int_{B_{\varepsilon}} \delta_{\varepsilon}^{8}\left(\Pi v_{\varepsilon}^{o}\right)^{2}+O\left(\frac{\left\|v_{\varepsilon}\right\|\left\|\Pi v_{\varepsilon}^{o}\right\|}{\left(\lambda_{\varepsilon} d_{\varepsilon}\right)^{1 / 2}}\right)
$$

Secondly, we write

$$
\int_{B_{\varepsilon}} P \delta_{\varepsilon}^{9-\varepsilon} \Pi v_{\varepsilon}^{o}=\int_{B_{\varepsilon}} \delta_{\varepsilon}^{9-\varepsilon} \Pi v_{\varepsilon}^{o}-(9-\varepsilon) \int_{B_{\varepsilon}} \delta_{\varepsilon}^{8-\varepsilon} \theta_{\varepsilon} \Pi v_{\varepsilon}^{o}+O\left(\int_{B_{\varepsilon}} \delta_{\varepsilon}^{7-\varepsilon} \theta_{\varepsilon}^{2}\left|\Pi v_{\varepsilon}^{o}\right|\right)
$$

Thus, using the evenness of $\delta_{\varepsilon}$, the oddness of $\Pi v_{\varepsilon}^{o}$ and Holder inequality, we obtain

$$
\int_{B_{\varepsilon}} P \delta_{\varepsilon}^{9-\varepsilon} \Pi v_{\varepsilon}^{o}=O\left(\frac{\left\|\Pi v_{\varepsilon}^{o}\right\|}{\left(\lambda_{\varepsilon} d_{\varepsilon}\right)^{2}}\right)
$$

Using (2.54), (2.56), (2.58) and Propositions 2.2 and 2.4, we easily derive our lemma.

Lemma 2.11. For \& small, we have

$$
\left\|\Pi v_{\varepsilon}^{o}\right\|=O\left(\varepsilon^{3 / 2}+\frac{1}{\left(\lambda_{\varepsilon} d_{\varepsilon}\right)^{3 / 2}}\right) .
$$

Proof. We write

$$
\Pi v_{\varepsilon}^{o}=\widetilde{\Pi} v_{\varepsilon}^{o}+\alpha \Pi \delta_{\varepsilon}+\beta \lambda_{\varepsilon} \frac{\partial \Pi \delta_{\varepsilon}}{\partial \lambda}+\sum_{r=1}^{5} \gamma_{r} \frac{1}{\lambda_{\varepsilon}} \frac{\partial \Pi \delta_{\varepsilon}}{\partial a_{r}}
$$

with

$$
\left\langle\widetilde{\Pi} v_{\varepsilon}^{o}, \Pi \delta_{\varepsilon}\right\rangle=\left\langle\widetilde{\Pi} v_{\varepsilon}^{o}, \frac{\partial \Pi \delta_{\varepsilon}}{\partial \lambda}\right\rangle=\left\langle\widetilde{\Pi} v_{\varepsilon}^{o}, \frac{\partial \Pi \delta_{\varepsilon}}{\partial a_{r}}\right\rangle=0 \quad \text { for each } r \in\{1,2,3,4,5\}
$$


12 Single blow-up solutions for a biharmonic equation

Taking the scalar product in $H^{2} \cap H_{0}^{1}\left(B_{\varepsilon}\right)$ of (2.60) with $\Pi \delta_{\varepsilon}, \lambda_{\varepsilon} \partial \Pi \delta_{\varepsilon} / \partial \lambda, \lambda_{\varepsilon}^{-1} \partial \Pi \delta_{\varepsilon} / \partial a_{r}, 1 \leq$ $r \leq 5$, provides us with the following invertible linear system in $\alpha, \beta, \gamma_{r}$ (with $1 \leq r \leq 5$ )

$$
\begin{aligned}
\left\langle\Pi \delta_{\varepsilon}, \Pi v_{\varepsilon}^{o}\right\rangle= & \alpha\left(C^{\prime}+o(1)\right)+\beta\left\langle\Pi \delta_{\varepsilon}, \lambda_{\varepsilon} \frac{\partial \Pi \delta_{\varepsilon}}{\partial \lambda}\right\rangle+\sum_{r=1}^{5} \gamma_{r}\left\langle\Pi \delta_{\varepsilon}, \frac{1}{\lambda_{\varepsilon}} \frac{\partial \Pi \delta_{\varepsilon}}{\partial a_{r}}\right\rangle \\
\left\langle\lambda_{\varepsilon} \frac{\partial \Pi \delta_{\varepsilon}}{\partial \lambda}, \Pi v_{\varepsilon}^{o}\right\rangle= & \alpha\left\langle\Pi \delta_{\varepsilon}, \lambda_{\varepsilon} \frac{\partial \Pi \delta_{\varepsilon}}{\partial \lambda}\right\rangle+\beta\left(C^{\prime \prime}+o(1)\right)+\sum_{r=1}^{5} \gamma_{r}\left\langle\lambda_{\varepsilon} \frac{\partial \Pi \delta_{\varepsilon}}{\partial \lambda}, \frac{1}{\lambda_{\varepsilon}} \frac{\partial \Pi \delta_{\varepsilon}}{\partial a_{r}}\right\rangle \\
\left\langle\frac{1}{\lambda_{\varepsilon}} \frac{\partial \Pi \delta_{\varepsilon}}{\partial a_{k}}, \Pi v_{\varepsilon}^{o}\right\rangle= & \alpha\left\langle\Pi \delta_{\varepsilon}, \frac{1}{\lambda_{\varepsilon}} \frac{\partial \Pi \delta_{\varepsilon}}{\partial a_{k}}\right\rangle+\beta\left\langle\lambda_{\varepsilon} \frac{\partial \Pi \delta_{\varepsilon}}{\partial \lambda}, \frac{1}{\lambda_{\varepsilon}} \frac{\partial \Pi \delta_{\varepsilon}}{\partial a_{k}}\right\rangle \\
& +\sum_{r=1}^{5} \gamma_{r}\left\langle\frac{1}{\lambda_{\varepsilon}} \frac{\partial \Pi \delta_{\varepsilon}}{\partial a_{k}}, \frac{1}{\lambda_{\varepsilon}} \frac{\partial \Pi \delta_{\varepsilon}}{\partial a_{r}}\right\rangle .
\end{aligned}
$$

Observe that

$$
\begin{gathered}
\left\langle\Pi \delta_{\varepsilon}, \lambda_{\varepsilon} \frac{\partial \Pi \delta_{\varepsilon}}{\partial \lambda}\right\rangle=O\left(\frac{1}{\lambda_{\varepsilon} d_{\varepsilon}}\right) ; \\
\left\langle\lambda_{\varepsilon} \frac{\partial \Pi \delta_{\varepsilon}}{\partial \lambda}, \frac{1}{\lambda_{\varepsilon}} \frac{\partial \Pi \delta_{\varepsilon}}{\partial a_{r}}\right\rangle=O\left(\frac{1}{\left(\lambda_{\varepsilon} d_{\varepsilon}\right)^{2}}\right) ; \\
\left\langle\Pi \delta_{\varepsilon}, \frac{1}{\lambda_{\varepsilon}} \frac{\partial \Pi \delta_{\varepsilon}}{\partial a_{r}}\right\rangle=O\left(\frac{1}{\left(\lambda_{\varepsilon} d_{\varepsilon}\right)^{2}}\right) ; \\
\left\langle\frac{1}{\lambda_{\varepsilon}} \frac{\partial \Pi \delta_{\varepsilon}}{\partial a_{k}}, \frac{1}{\lambda_{\varepsilon}} \frac{\partial \Pi \delta_{\varepsilon}}{\partial a_{r}}\right\rangle=\left(C^{\prime \prime \prime}+o(1)\right) \delta_{k r}+O\left(\frac{1}{\left(\lambda_{\varepsilon} d_{\varepsilon}\right)^{2}}\right)
\end{gathered}
$$

where $\delta_{k r}$ denotes the Kronecker symbol.

Now, because of the evenness of $\delta_{\varepsilon}$ and the oddness of $\Pi v_{\varepsilon}^{o}$ with respect to $\left(x-a_{\varepsilon}\right)_{k}$ we obtain

$$
\left\langle\Pi \delta_{\varepsilon}, \Pi v_{\varepsilon}^{o}\right\rangle=\int_{B_{\varepsilon}} \Delta \Pi \delta_{\varepsilon} \cdot \Delta \Pi v_{\varepsilon}^{o}=\int_{B_{\varepsilon}} \delta_{\varepsilon}^{9} \Pi v_{\varepsilon}^{o}=0
$$

In the same way we have

$$
\left\langle\lambda_{\varepsilon} \frac{\partial \Pi \delta_{\varepsilon}}{\partial \lambda}, \Pi v_{\varepsilon}^{o}\right\rangle=\left\langle\frac{1}{\lambda_{\varepsilon}} \frac{\partial \Pi \delta_{\varepsilon}}{\partial a_{r}}, \Pi v_{\varepsilon}^{o}\right\rangle=0 \quad \text { for each } r \neq k
$$

We also have

$$
\begin{aligned}
\left\langle\frac{1}{\lambda_{\varepsilon}} \frac{\partial \Pi \delta_{\varepsilon}}{\partial a_{k}}, \Pi v_{\varepsilon}^{o}\right\rangle & =\int_{B_{\varepsilon}} \Delta\left(\frac{1}{\lambda_{\varepsilon}} \frac{\partial \Pi \delta_{\varepsilon}}{\partial a_{k}}\right) \cdot \Delta\left(v_{\varepsilon}-\Pi v_{\varepsilon}^{e}-w_{\varepsilon}\right) \\
& =\int_{B_{\varepsilon}} \Delta\left(\frac{1}{\lambda_{\varepsilon}} \frac{\partial \Pi \delta_{\varepsilon}}{\partial a_{k}}\right) \cdot \Delta v_{\varepsilon}-\int_{B_{\varepsilon}} \Delta\left(\frac{1}{\lambda_{\varepsilon}} \frac{\partial \Pi \delta_{\varepsilon}}{\partial a_{k}}\right) \cdot \Delta w_{\varepsilon},
\end{aligned}
$$


where we have used in the last equality the fact that $\Pi v_{\varepsilon}^{e}$ is even with respect to $\left(x-a_{\varepsilon}\right)_{k}$. Using (2.37) and Holder inequality, we obtain

$$
\int_{B_{\varepsilon}} \Delta\left(\frac{1}{\lambda_{\varepsilon}} \frac{\partial \Pi \delta_{\varepsilon}}{\partial a_{k}}\right) \cdot \Delta v_{\varepsilon} \leq c\left\|v_{\varepsilon}\right\|\left(\int_{\Omega \backslash B_{\varepsilon}}\left|\Delta \frac{1}{\lambda_{\varepsilon}} \frac{\partial \delta_{\varepsilon}}{\partial a_{k}}\right|^{2}\right)^{1 / 2}=O\left(\frac{\left\|v_{\varepsilon}\right\|}{\left(\lambda_{\varepsilon} d_{\varepsilon}\right)^{3 / 2}}\right) .
$$

Equation (2.66) and Lemma 2.7 imply that

$$
\left\langle\frac{1}{\lambda_{\varepsilon}} \frac{\partial \Pi \delta_{\varepsilon}}{\partial a_{k}}, \Pi v_{\varepsilon}^{o}\right\rangle=O\left(\frac{\left\|v_{\varepsilon}\right\|}{\left(\lambda_{\varepsilon} d_{\varepsilon}\right)^{3 / 2}}\right)
$$

Inverting the linear system $(\mathrm{S})$, we deduce from the above estimates

$$
\begin{array}{ll}
\alpha=O\left(\frac{\left\|v_{\varepsilon}\right\|}{\left(\lambda_{\varepsilon} d_{\varepsilon}\right)^{7 / 2}}\right), & \beta=O\left(\frac{\left\|v_{\varepsilon}\right\|}{\left(\lambda_{\varepsilon} d_{\varepsilon}\right)^{7 / 2}}\right), \\
\gamma_{k}=O\left(\frac{\left\|v_{\varepsilon}\right\|}{\left(\lambda_{\varepsilon} d_{\varepsilon}\right)^{3 / 2}}\right), & \gamma_{r}=O\left(\frac{\left\|v_{\varepsilon}\right\|}{\left(\lambda_{\varepsilon} d_{\varepsilon}\right)^{7 / 2}}\right), \quad r \neq k .
\end{array}
$$

This implies through (2.60)

$$
\left\|\Pi v_{\varepsilon}^{o}-\tilde{\Pi} v_{\varepsilon}^{o}\right\|=O\left(\frac{\left\|v_{\varepsilon}\right\|}{\left(\lambda_{\varepsilon} d_{\varepsilon}\right)^{3 / 2}}\right), \quad\left\|\Pi v_{\varepsilon}^{o}\right\|^{2}=\left\|\tilde{\Pi} v_{\varepsilon}^{o}\right\|^{2}+O\left(\frac{\left\|v_{\varepsilon}\right\|^{2}}{\left(\lambda_{\varepsilon} d_{\varepsilon}\right)^{3}}\right) \text {. }
$$

We now turn to the last step, which consists in estimating $\left\|\tilde{\Pi} v_{\varepsilon}^{o}\right\|$. Since $u_{\varepsilon}$ is a solution of $\left(P_{\varepsilon}\right)$, we have

$$
\int_{B_{\varepsilon}} \Delta^{2} u_{\varepsilon} \Pi v_{\varepsilon}^{o}=\int_{B_{\varepsilon}} u_{\varepsilon}^{9-\varepsilon} \Pi v_{\varepsilon}^{o}
$$

Because of the evenness of $\delta_{\varepsilon}$ and the oddness of $\Pi v_{\varepsilon}^{o}$ with respect to $\left(x-a_{\varepsilon}\right)_{k},(2.70)$ becomes

$$
\left\|\Pi v_{\varepsilon}^{o}\right\|^{2}=\int_{B_{\varepsilon}} u_{\varepsilon}^{9-\varepsilon} \Pi v_{\varepsilon}^{o}
$$

By (2.69), (2.71) and Lemma 2.10, we obtain

$$
\left\|\widetilde{\Pi v_{\varepsilon}^{o}}\right\|^{2}-9 \int_{B_{\varepsilon}} \delta_{\varepsilon}^{8}\left(\Pi v_{\varepsilon}^{o}\right)^{2}+o\left(\left\|\widetilde{\Pi v_{\varepsilon}^{o}}\right\|^{2}\right)=O\left(\varepsilon^{3}+\frac{1}{\left(\lambda_{\varepsilon} d_{\varepsilon}\right)^{3}}\right)
$$

Using now (2.72) and the fact that the quadratic form

$$
v \longmapsto \int_{B_{\varepsilon}}|\Delta v|^{2}-9 \int_{B_{\varepsilon}} \delta_{i}^{8} v^{2}
$$


14 Single blow-up solutions for a biharmonic equation

is positive definite (see [6]) on the subset $\left[\operatorname{Span}\left(\Pi \delta_{\varepsilon}, \partial \Pi \delta_{\varepsilon} / \partial \lambda, \partial \Pi \delta_{\varepsilon} / \partial a_{k} \quad 1 \leq k \leq\right.\right.$ 5) $]_{H^{2} \cap H_{0}^{1}\left(B_{\varepsilon}\right)}^{\perp}$, we obtain

$$
\left\|\widetilde{\Pi v_{\varepsilon}^{o}}\right\| \leq C\left(\frac{1}{\left(\lambda_{\varepsilon} d_{\varepsilon}\right)^{3 / 2}}+\varepsilon^{3 / 2}\right) .
$$

Our lemma follows from (2.69) and (2.74).

Before ending this section, let us prove the following estimate which will be needed later.

LeMma 2.12. For \& small, we have

$$
\left\langle\frac{\partial^{2} P \delta_{\varepsilon}}{\partial \lambda \partial a_{k}}, v_{\varepsilon}\right\rangle=O\left(\frac{1}{\left(\lambda_{\varepsilon} d_{\varepsilon}\right)^{3 / 2}}+\varepsilon^{3 / 2}\right) .
$$

Proof. We have

$$
\begin{aligned}
\int_{\Omega} \Delta\left(\frac{\partial^{2} P \delta_{\varepsilon}}{\partial \lambda \partial a_{k}}\right) \Delta v_{\varepsilon} & =\int_{B_{\varepsilon}} \Delta^{2}\left(\frac{\partial^{2} P \delta_{\varepsilon}}{\partial \lambda \partial a_{k}}\right) v_{\varepsilon}+O\left(\frac{\left\|v_{\varepsilon}\right\|}{\left(\lambda_{\varepsilon} d_{\varepsilon}\right)^{9 / 2}}\right) \\
& =\int_{B_{\varepsilon}} \Delta^{2}\left(\frac{\partial^{2} P \delta_{\varepsilon}}{\partial \lambda \partial a_{k}}\right) \Pi v_{\varepsilon}^{o}+\int_{B_{\varepsilon}} \Delta^{2}\left(\frac{\partial^{2} P \delta_{\varepsilon}}{\partial \lambda \partial a_{k}}\right) w_{\varepsilon}+O\left(\frac{\left\|v_{\varepsilon}\right\|}{\left(\lambda_{\varepsilon} d_{\varepsilon}\right)^{9 / 2}}\right) .
\end{aligned}
$$

For the first integral on the right-hand side in (2.76), we have

$$
\int_{B_{\varepsilon}} \Delta^{2}\left(\frac{\partial^{2} P \delta_{\varepsilon}}{\partial \lambda \partial a_{k}}\right) \Pi v_{\varepsilon}^{o}=O\left(\left\|\Pi v_{\varepsilon}^{o}\right\|\right)=O\left(\frac{1}{\left(\lambda_{\varepsilon} d_{\varepsilon}\right)^{3 / 2}}+\varepsilon^{3 / 2}\right),
$$

where we have used in the last equality Lemma 2.11 .

Now let $\psi_{4}$ be the solution of

$$
\Delta^{2} \psi_{4}=\Delta^{2}\left(\frac{\partial^{2} P \delta_{\varepsilon}}{\partial \lambda \partial a_{k}}\right) \quad \text { in } B_{\varepsilon}, \quad \Delta \psi_{4}=\psi_{4}=0 \quad \text { on } \partial B_{\varepsilon} .
$$

Thus, as in the proof of Lemma 2.6, we obtain for $y \in \partial B_{\varepsilon}$

$$
\frac{\partial \psi_{4}}{\partial \nu}(y)=O\left(\frac{1}{\lambda_{\varepsilon}^{1 / 2} d_{\varepsilon}^{2}}\right), \quad \frac{\partial \Delta \psi_{4}}{\partial \nu}(y)=O\left(\frac{1}{\lambda_{\varepsilon}^{1 / 2} d_{\varepsilon}^{4}}\right)
$$

and therefore

$$
\int_{B_{\varepsilon}} \Delta^{2}\left(\frac{\partial^{2} P \delta_{\varepsilon}}{\partial \lambda \partial a_{k}}\right) w_{\varepsilon}=O\left(\frac{\left\|v_{\varepsilon}\right\|}{\left(\lambda_{\varepsilon} d_{\varepsilon}\right)^{1 / 2}}\right)
$$

From (2.76), (2.77), (2.80) and Proposition 2.4, we easily deduce our lemma. 


\section{Proof of theorems}

Let us start by proving the following crucial result.

Proposition 3.1. For $u_{\varepsilon}=\alpha_{\varepsilon} P \delta_{a_{\varepsilon}, \lambda_{\varepsilon}}+v_{\varepsilon}$ solution of $\left(P_{\varepsilon}\right)$ with $\lambda_{\varepsilon}^{\varepsilon}=1+o(1)$ as $\varepsilon$ goes to zero, we have the following estimates

(a) $c_{2} \varepsilon+O\left(\varepsilon^{2}\right)-c_{1}\left(H\left(a_{\varepsilon}, a_{\varepsilon}\right) / \lambda_{\varepsilon}\right)+o\left(1 / \lambda_{\varepsilon} d_{\varepsilon}\right)=0$,

(b) $\left(c_{3} / \lambda_{\varepsilon}^{2}\right)\left(\partial H\left(a_{\varepsilon}, a_{\varepsilon}\right) / \partial a\right)+o\left(1 /\left(\lambda_{\varepsilon} d_{\varepsilon}\right)^{2}\right)+O\left(\varepsilon^{5 / 2}+\varepsilon \log \left(\lambda_{\varepsilon}\right) /\left(\lambda_{\varepsilon} d_{\varepsilon}\right)^{2}+1 /\left(\lambda_{\varepsilon} d_{\varepsilon}\right)^{5 / 2}\right)=0$,

where $c_{1}, c_{2}$ are the constants defined in Theorem 1.1, and where $c_{3}>0$.

Proof. Since claim (a) was proved in [5], we only need to prove claim (b). Multiplying $\left(P_{\varepsilon}\right)$ by $\left(1 / \lambda_{\varepsilon}\right)\left(\partial P \delta_{\varepsilon} / \partial a_{k}\right)$ and integrating on $\Omega$, we obtain for $1 \leq k \leq 5$

$$
\begin{aligned}
0=\int_{\Omega} \Delta^{2} u_{\varepsilon} \frac{1}{\lambda_{\varepsilon}} \frac{\partial P \delta_{\varepsilon}}{\partial a_{k}}-\int_{\Omega} u_{\varepsilon}^{9-\varepsilon} \frac{1}{\lambda_{\varepsilon}} \frac{\partial P \delta_{\varepsilon}}{\partial a_{k}} & \\
=\alpha_{\varepsilon} \int_{\Omega} \delta_{\varepsilon}^{9} \frac{1}{\lambda_{\varepsilon}} \frac{\partial P \delta_{\varepsilon}}{\partial a_{k}}-\int_{\Omega}[ & \left(\alpha_{\varepsilon} P \delta_{\varepsilon}\right)^{9-\varepsilon}+(9-\varepsilon)\left(\alpha_{\varepsilon} P \delta_{\varepsilon}\right)^{8-\varepsilon} v_{\varepsilon} \\
& \left.\quad+\frac{(9-\varepsilon)(8-\varepsilon)}{2}\left(\alpha_{\varepsilon} P \delta_{\varepsilon}\right)^{7-\varepsilon} v_{\varepsilon}^{2}\right] \frac{1}{\lambda_{\varepsilon}} \frac{\partial P \delta_{\varepsilon}}{\partial a_{k}}+O\left(\left\|v_{\varepsilon}\right\|^{3}\right) .
\end{aligned}
$$

We estimate each term on the right-hand side in (3.1). First, by Proposition 2.1 and the Holder inequality, we have

$$
\int_{\Omega} P \delta_{\varepsilon}^{7-\varepsilon} v_{\varepsilon}^{2} \frac{1}{\lambda_{\varepsilon}} \frac{\partial P \delta_{\varepsilon}}{\partial a_{k}}=\int_{\Omega} \delta_{\varepsilon}^{7-\varepsilon} v_{\varepsilon}^{2} \frac{1}{\lambda_{\varepsilon}} \frac{\partial \delta_{\varepsilon}}{\partial a_{k}}+O\left(\frac{\left\|v_{\varepsilon}\right\|^{2}}{\lambda_{\varepsilon} d_{\varepsilon}}\right) .
$$

Secondly, we compute

$$
\begin{aligned}
\int_{\Omega} P \delta_{\varepsilon}^{8-\varepsilon} v_{\varepsilon} \frac{1}{\lambda_{\varepsilon}} \frac{\partial P \delta_{\varepsilon}}{\partial a_{k}}= & \int_{\Omega} \delta_{\varepsilon}^{8-\varepsilon} v_{\varepsilon} \frac{1}{\lambda_{\varepsilon}} \frac{\partial P \delta_{\varepsilon}}{\partial a_{k}}+(8-\varepsilon) \int_{\Omega} \delta_{\varepsilon}^{7-\varepsilon} \theta_{\varepsilon} v_{\varepsilon} \frac{1}{\lambda_{\varepsilon}} \frac{\partial P \delta_{\varepsilon}}{\partial a_{k}}+O\left(\int_{\Omega} \delta_{\varepsilon}^{7-\varepsilon} \theta_{\varepsilon}^{2}\left|v_{\varepsilon}\right|\right) \\
= & \int_{\Omega} \delta_{\varepsilon}^{8-\varepsilon} v_{\varepsilon} \frac{1}{\lambda_{\varepsilon}} \frac{\partial \delta_{\varepsilon}}{\partial a_{k}}+O\left(\int_{\Omega} \delta_{\varepsilon}^{8-\varepsilon}\left|v_{\varepsilon}\right|\left|\frac{1}{\lambda_{\varepsilon}} \frac{\partial \theta_{\varepsilon}}{\partial a_{k}}\right|\right) \\
& +(8-\varepsilon) \int_{\Omega} \delta_{\varepsilon}^{7-\varepsilon} \theta_{\varepsilon} v_{\varepsilon} \frac{1}{\lambda_{\varepsilon}} \frac{\partial \delta_{\varepsilon}}{\partial a_{k}} \\
& +O\left(\int_{\Omega} \delta_{\varepsilon}^{7-\varepsilon} \theta_{\varepsilon}\left|v_{\varepsilon}\right|\left|\frac{1}{\lambda_{\varepsilon}} \frac{\partial \theta_{\varepsilon}}{\partial a_{k}}\right|\right)+O\left(\int_{\Omega} \delta_{\varepsilon}^{7-\varepsilon} \theta_{\varepsilon}^{2}\left|v_{\varepsilon}\right|\right)
\end{aligned}
$$

By Proposition 2.1 and the Holder inequality, we obtain

$$
\begin{gathered}
\int_{\Omega} \delta_{\varepsilon}^{7-\varepsilon} \theta_{\varepsilon}^{2}\left|v_{\varepsilon}\right|=O\left(\frac{\left\|v_{\varepsilon}\right\|}{\left(\lambda_{\varepsilon} d_{\varepsilon}\right)^{2}}\right), \quad \int_{\Omega} \delta_{\varepsilon}^{7-\varepsilon} \theta_{\varepsilon}\left|v_{\varepsilon}\right|\left|\frac{1}{\lambda_{\varepsilon}} \frac{\partial \theta_{\varepsilon}}{\partial a_{k}}\right|=O\left(\frac{\left\|v_{\varepsilon}\right\|}{\left(\lambda_{\varepsilon} d_{\varepsilon}\right)^{3}}\right), \\
\int_{\Omega} \delta_{\varepsilon}^{8-\varepsilon}\left|v_{\varepsilon}\right|\left|\frac{1}{\lambda_{\varepsilon}} \frac{\partial \theta_{\varepsilon}}{\partial a_{k}}\right|=O\left(\frac{\left\|v_{\varepsilon}\right\|}{\left(\lambda_{\varepsilon} d_{\varepsilon}\right)^{2}}\right) .
\end{gathered}
$$


16 Single blow-up solutions for a biharmonic equation

We also have by Proposition 2.2

$$
\begin{aligned}
\int_{\Omega} \delta_{\varepsilon}^{8-\varepsilon} v_{\varepsilon} \frac{1}{\lambda_{\varepsilon}} \frac{\partial \delta_{\varepsilon}}{\partial a_{k}} & =\int_{\Omega} \delta_{\varepsilon}^{8}\left(\delta_{\varepsilon}^{-\varepsilon}-\frac{c_{0}^{-\varepsilon}}{\lambda_{\varepsilon}^{\varepsilon / 2}}\right) v_{\varepsilon} \frac{1}{\lambda_{\varepsilon}} \frac{\partial \delta_{\varepsilon}}{\partial a_{k}} \\
& =\int_{B_{\varepsilon}} \delta_{\varepsilon}^{8}\left(\delta_{\varepsilon}^{-\varepsilon}-\frac{c_{0}^{-\varepsilon}}{\lambda_{\varepsilon}^{\varepsilon / 2}}\right) v_{\varepsilon} \frac{1}{\lambda_{\varepsilon}} \frac{\partial \delta_{\varepsilon}}{\partial a_{k}}+O\left(\frac{\left\|v_{\varepsilon}\right\|}{\left(\lambda_{\varepsilon} d_{\varepsilon}\right)^{9 / 2}}\right) .
\end{aligned}
$$

Using (2.17), Lemma 2.3 and the Holder inequality, we derive that

$$
\begin{aligned}
\int_{\Omega} \delta_{\varepsilon}^{8-\varepsilon} v_{\varepsilon} \frac{1}{\lambda_{\varepsilon}} \frac{\partial \delta_{\varepsilon}}{\partial a_{k}} & =\int_{B_{\varepsilon}} \delta_{\varepsilon}^{8}\left(\delta_{\varepsilon}^{-\varepsilon}-\frac{c_{0}^{-\varepsilon}}{\lambda_{\varepsilon}^{\varepsilon / 2}}\right) \frac{1}{\lambda_{\varepsilon}} \frac{\partial \delta_{\varepsilon}}{\partial a_{k}} w_{\varepsilon}+O\left(\varepsilon\left\|\Pi v_{\varepsilon}^{o}\right\|+\frac{\left\|v_{\varepsilon}\right\|}{\left(\lambda_{\varepsilon} d_{\varepsilon}\right)^{9 / 2}}\right) \\
& =O\left(\frac{\varepsilon\left\|v_{\varepsilon}\right\|}{\left(\lambda_{\varepsilon} d_{\varepsilon}\right)^{1 / 2}}+\varepsilon\left\|\Pi v_{\varepsilon}^{o}\right\|+\frac{\left\|v_{\varepsilon}\right\|}{\left(\lambda_{\varepsilon} d_{\varepsilon}\right)^{9 / 2}}\right),
\end{aligned}
$$

where we have used Lemma 2.6 in the last equality.

Using (3.2)-(3.6), Lemmas 2.5, 2.8, 2.9, Proposition 2.2 and the fact that $\lambda_{\varepsilon}^{\varepsilon}=1+$ $O\left(\varepsilon \log \lambda_{\varepsilon}\right)$, we easily derive our result.

We are now able to prove Theorem 1.1.

Proof of Theorem 1.1. Let $\left(u_{\varepsilon}\right)$ be a solution of $\left(P_{\varepsilon}\right)$ which satisfies $(H)$. Then, using Proposition 2.2, $u_{\varepsilon}=\alpha_{\varepsilon} P \delta_{a_{\varepsilon}, \lambda_{\varepsilon}}+v_{\varepsilon}$ with $\alpha_{\varepsilon} \rightarrow 1, \lambda_{\varepsilon}^{\varepsilon} \rightarrow 1, \lambda_{\varepsilon} d\left(a_{\varepsilon}, \partial \Omega\right) \rightarrow \infty, v_{\varepsilon}$ satisfies $\left(V_{0}\right)$ and $\left\|v_{\varepsilon}\right\| \rightarrow 0$. Now, using claim (a) of Proposition 3.1, we derive that

$$
\varepsilon=\frac{c_{1}}{c_{2}} \frac{H\left(a_{\varepsilon}, a_{\varepsilon}\right)}{\lambda_{\varepsilon}}+o\left(\frac{1}{\lambda_{\varepsilon} d_{\varepsilon}}\right)=O\left(\frac{1}{\lambda_{\varepsilon} d_{\varepsilon}}\right) .
$$

Therefore, it follows from claim (b) of Proposition 3.1 that

$$
\frac{\partial H\left(a_{\varepsilon}, a_{\varepsilon}\right)}{\partial a}=o\left(\frac{1}{d_{\varepsilon}^{2}}\right) .
$$

Using (3.8) and the fact that for $a$ near the boundary $(\partial H / \partial a)\left(a_{\varepsilon}, a_{\varepsilon}\right) \sim c d\left(a_{\varepsilon}, \partial \Omega\right)^{-2}$, we derive that $a_{\varepsilon}$ is away from the boundary and it converges to a critical point $x_{0}$ of $\varphi$.

Finally, using (3.7), we obtain

$$
\varepsilon \lambda_{\varepsilon} \longrightarrow \frac{c_{1}}{c_{2}} \varphi\left(x_{0}\right) \quad \text { as } \varepsilon \longrightarrow 0
$$

By Proposition 2.2, we have

$$
\left\|u_{\varepsilon}\right\|_{L^{\infty}}^{2} \sim c_{0}^{2} \lambda_{\varepsilon} \quad \text { as } \varepsilon \longrightarrow 0 .
$$

This concludes the proof of Theorem 1.1.

The sequel of this section is devoted to the proof of Theorem 1.2. 
Proof of Theorem 1.2. Let $x_{0}$ be a nondegenerate critical point of $\varphi$. It is easy to see that $d(a, \partial \Omega)>d_{0}>0$ for $a$ near $x_{0}$. We will take a function $u=\alpha P \delta_{(a, \lambda)}+v$ where $\left(\alpha-\alpha_{0}\right)$ is very small, $\lambda$ is large enough, $\|v\|$ is very small, $a$ is close to $x_{0}$ and $\alpha_{0}=S^{-5 / 8}$ and we will prove that we can choose the variables $(\alpha, \lambda, a, v)$ so that $u$ is a critical point of $J_{\varepsilon}$ with $\|u\|=1$. Here $J_{\varepsilon}$ denotes the functional corresponding to problem $\left(P_{\varepsilon}\right)$ defined by

$$
J_{\varepsilon}(u)=\left(\int_{\Omega}|\Delta u|^{2}\right)\left(\int_{\Omega}|u|^{10-\varepsilon}\right)^{-2 /(10-\varepsilon)} .
$$

Let

$$
\begin{aligned}
& M_{\varepsilon}=\left\{(\alpha, \lambda, a, v) \in \mathbb{R}_{+}^{*} \times \mathbb{R}_{+}^{*} \times \Omega \times \mathscr{H}(\Omega) /\left|\alpha-\alpha_{0}\right|<\nu_{0},\right. \\
&\left.d_{a}>d_{0}, \lambda>v_{0}^{-1}, \varepsilon \log \lambda<\nu_{0},\|v\|<\nu_{0} \text { and } v \in E_{(a, \lambda)}\right\},
\end{aligned}
$$

where $\nu_{0}$ and $d_{0}$ are two suitable positive constants and where $d_{a}=d(a, \partial \Omega)$.

Let us define the functional

$$
K_{\varepsilon}: M_{\varepsilon} \longrightarrow \mathbb{R}, \quad K_{\varepsilon}(\alpha, a, \lambda, v)=J_{\varepsilon}\left(\alpha P \delta_{(a, \lambda)}+v\right) .
$$

It is known that $(\alpha, \lambda, a, v)$ is a critical point of $K_{\varepsilon}$ if and only if $u=\alpha P \delta_{(a, \lambda)}+v$ is a critical point of $J_{\varepsilon}$ on $\mathscr{H}(\Omega)$. So this fact allows us to look for critical points of $J_{\varepsilon}$ by successive optimizations with respect to the different parameters on $M_{\varepsilon}$.

First, arguing as in [25, Proposition 4] we see that the following problem

$$
\min \left\{J_{\mathcal{\varepsilon}}\left(\alpha P \delta_{(a, \lambda)}+v\right), v \text { satisfying }\left(V_{0}\right) \text { and }\|v\|<v_{0}\right\}
$$

is achieved by a unique function $\bar{v}$ which satisfies the estimate of Proposition 2.4. This implies that there exist $A, B$ and $C_{i}$ 's such that

$$
\frac{\partial K_{\varepsilon}}{\partial v}(\alpha, \lambda, a, \bar{v})=\nabla J_{\varepsilon}\left(\alpha P \delta_{(a, \lambda)}+\bar{v}\right)=A P \delta_{(a, \lambda)}+B \frac{\partial}{\partial \lambda} P \delta_{(a, \lambda)}+\sum_{i=1}^{5} C_{i} \frac{\partial}{\partial a_{i}} P \delta_{(a, \lambda)},
$$

where $a_{i}$ is the $i$ th component of $a$.

According to [5], we have that

$$
A=O\left(\varepsilon \log \lambda+|\beta|+\frac{1}{\lambda}\right), \quad B=O(\lambda \varepsilon+1), \quad C_{j}=O\left(\frac{\varepsilon^{2}}{\lambda}+\frac{1}{\lambda^{3}}\right) .
$$

To find critical points of $K_{\varepsilon}$, we have to solve the following system

$$
\begin{gathered}
\frac{\partial K_{\varepsilon}}{\partial \alpha}=0 \\
\frac{\partial K_{\varepsilon}}{\partial \lambda}=B\left\langle\frac{\partial^{2} P \delta}{\partial \lambda^{2}}, \bar{v}\right\rangle+\sum_{i=1}^{5} C_{i}\left\langle\frac{\partial^{2} P \delta}{\partial \lambda \partial a_{i}}, \bar{v}\right\rangle \\
\frac{\partial K_{\varepsilon}}{\partial a_{j}}=B\left\langle\frac{\partial^{2} P \delta}{\partial \lambda \partial a_{j}}, \bar{v}\right\rangle+\sum_{i=1}^{5} C_{i}\left\langle\frac{\partial^{2} P \delta}{\partial a_{i} \partial a_{j}}, \bar{v}\right\rangle, \quad \text { for each } j=1, \ldots, 5 .
\end{gathered}
$$


18 Single blow-up solutions for a biharmonic equation

Observe that for $\psi=P \delta_{(a, \lambda)}, \partial P \delta_{(a, \lambda)} / \partial \lambda, \partial P \delta_{(a, \lambda)} / \partial a_{i}$ with $i=1, \ldots, 5$ and for $u=\alpha P \delta_{(a, \lambda)}+$ $\bar{v}$, we have

$$
\left\langle\nabla J_{\mathcal{\varepsilon}}(u), \psi\right\rangle=2 J_{\mathcal{\varepsilon}}(u)\left(\alpha\left\langle P \delta_{(a, \lambda)}, \psi\right\rangle-J_{\mathcal{\varepsilon}}(u)^{5-\varepsilon / 2} \int_{\Omega}|u|^{8-\varepsilon} u \psi\right) .
$$

We also have (see [5])

$$
\begin{gathered}
J_{\varepsilon}\left(\alpha P \delta_{(a, \lambda)}+\bar{v}\right)=S+O\left(\varepsilon \log \lambda+\frac{1}{\lambda}\right) \\
\frac{\partial K_{\varepsilon}}{\partial \alpha}=\left\langle\nabla J_{\varepsilon}(\alpha P \delta+\bar{v}), P \delta\right\rangle=2 J_{\varepsilon}(u)\left(\alpha S^{5 / 4}\left(1-\alpha^{8} S^{5}\right)+O\left(\varepsilon \log \lambda+\frac{1}{\lambda}\right)\right) \\
\lambda \frac{\partial K_{\varepsilon}}{\partial \lambda}=\left\langle\nabla J_{\varepsilon}(\alpha P \delta+\bar{v}), \lambda \frac{\partial P \delta}{\partial \lambda}\right\rangle=J_{\varepsilon}(u)\left(\alpha c_{1} \frac{H(a, a)}{\lambda}\left(1-2 \alpha^{8} S^{5}\right)+c_{2} S^{5} \alpha^{9} \varepsilon\right. \\
\left.+O\left(\varepsilon^{2} \log \lambda+\frac{\varepsilon \log \lambda}{\lambda}+\frac{1}{\lambda^{3}}\right)\right)
\end{gathered}
$$

Following the proof of claim (b) of Proposition 3.1, we obtain, for each $j=1, \ldots, 5$,

$$
\frac{1}{\lambda} \frac{\partial K_{\varepsilon}}{\partial a_{j}}=\left\langle\nabla J_{\varepsilon}(\alpha P \delta+\bar{v}), \frac{1}{\lambda} \frac{\partial P \delta}{\partial a_{j}}\right\rangle=-\frac{c \alpha}{2 \lambda^{2}} \frac{\partial H(a, a)}{\partial a}\left(1-2 \alpha^{8} S^{5}\right)+O\left(\varepsilon^{5 / 2}+\frac{\varepsilon \log \lambda}{\lambda^{2}}+\frac{1}{\lambda^{5 / 2}}\right) .
$$

On the other hand, one can easily verify that

$$
\left\|\frac{\partial^{2} P \delta}{\partial \lambda^{2}}\right\|=O\left(\frac{1}{\lambda^{2}}\right), \quad\left\|\frac{\partial^{2} P \delta}{\partial a_{i} \partial a_{j}}\right\|=O\left(\lambda^{2}\right)
$$

Now, we take the following change of variables:

$$
\alpha=\alpha_{0}+\beta, \quad a=x_{0}+\xi, \quad \frac{1}{\lambda^{1 / 2}}=\sqrt{\frac{c_{2}}{c_{1}}}\left(\frac{1}{\sqrt{H\left(x_{0}, x_{0}\right)}}+\rho\right) \sqrt{\varepsilon} .
$$

Then, using estimates (3.18)-(3.22), Lemma 2.12, Proposition 2.4 and the fact that $x_{0}$ is a nondegenerate critical point of $\varphi$, the system $\left(E_{1}\right)$ becomes

$$
\begin{aligned}
& \beta=O\left(\varepsilon|\log \varepsilon|+|\beta|^{2}\right) \\
& \rho=O\left(\varepsilon|\log \varepsilon|+|\beta|^{2}+|\xi|^{2}+\rho^{2}\right) \\
& \xi=O\left(|\beta|^{2}+|\xi|^{2}+\varepsilon^{1 / 2}\right) .
\end{aligned}
$$

Thus Brower's fixed point theorem shows that the system $\left(E_{2}\right)$ has a solution $\left(\beta_{\varepsilon}, \rho_{\varepsilon}, \xi_{\varepsilon}\right)$ for $\varepsilon$ small enough such that

$$
\beta_{\varepsilon}=O(\varepsilon|\log \varepsilon|), \quad \rho_{\varepsilon}=O(\varepsilon|\log \varepsilon|), \quad \xi_{\varepsilon}=O\left(\varepsilon^{1 / 2}\right)
$$


By construction, the corresponding $u_{\varepsilon}$ is a critical point of $J_{\varepsilon}$ that is $w_{\varepsilon}=J_{\varepsilon}\left(u_{\varepsilon}\right)^{(5-\varepsilon / 2) /(8-\varepsilon)} u_{\varepsilon}$ satisfies

$$
\Delta^{2} w_{\varepsilon}=\left|w_{\varepsilon}\right|^{8-\varepsilon} w_{\varepsilon} \quad \text { in } \Omega, w_{\varepsilon}=\Delta w_{\varepsilon}=0 \text { on } \partial \Omega
$$

with $\left|w_{\varepsilon}^{-}\right|_{L^{10}(\Omega)}$ very small, where $w_{\varepsilon}^{-}=\max \left(0,-w_{\varepsilon}\right)$.

As in [7, Proposition 4.1], we prove that $w_{\varepsilon}^{-}=0$. Thus, since $w_{\varepsilon}$ is a non-negative function which satisfies (3.25), the strong maximum principle ensures that $w_{\varepsilon}>0$ on $\Omega$ and then $w_{\varepsilon}$ is a solution of $\left(P_{\varepsilon}\right)$, which blows up at $x_{0}$ as $\varepsilon$ goes to zero. This ends the proof of Theorem 1.2.

\section{References}

[1] F. V. Atkinson and L. A. Peletier, Elliptic equations with nearly critical growth, Journal of Differential Equations 70 (1987), no. 3, 349-365.

[2] A. Bahri, Critical Points at Infinity in Some Variational Problems, Pitman Research Notes in Mathematics Series, vol. 182, Longman Scientific \& Technical, Harlow, 1989.

[3] A. Bahri, Y. Li, and O. Rey, On a variational problem with lack of compactness: the topological effect of the critical points at infinity, Calculus of Variations and Partial Differential Equations 3 (1995), no. 1, 67-93.

[4] M. Ben Ayed and K. El Mehdi, Existence of conformal metrics on spheres with prescribed Paneitz curvature, Manuscripta Mathematica 114 (2004), no. 2, 211-228.

[5] _ On a biharmonic equation involving nearly critical exponent, to appear in Nonlinear Differential Equations and Applications, 2006.

[6] - The Paneitz curvature problem on lower dimensional spheres, to appear in Annals of Global Analysis and Geometry.

[7] M. Ben Ayed, K. El Mehdi, and M. Hammami, Some existence results for a Paneitz type problem via the theory of critical points at infinity, Journal de Mathématiques Pures et Appliquées. Neuvième Série 84 (2005), no. 2, 247-278.

[8] M. Ben Ayed and M. Hammami, On a fourth order elliptic equation with critical nonlinearity in dimension six, to appear in Nonlinear Analysis TMA.

[9] H. Brezis and L. A. Peletier, Asymptotics for elliptic equations involving critical growth, Partial Differential Equations and the Calculus of Variations, Vol. 1, Progr. Nonlinear Differential Equations Appl., vol. 1, Birkhäuser Boston, Massachusetts, 1989, pp. 149-192.

[10] S.-Y. A. Chang, On a fourth-order partial differential equation in conformal geometry, Harmonic Analysis and Partial Differential Equations (Chicago, Ill, 1996) (M. Christ, C. Kenig, and C. Sadorsky, eds.), Chicago Lectures in Math., University of Chicago Press, Illinois, 1999, pp. 127150, essays in honor of Alberto P. Calderon.

[11] K.-S. Chou and D. Geng, Asymptotics of positive solutions for a biharmonic equation involving critical exponent, Differential and Integral Equations. An International Journal for Theory \&Applications 13 (2000), no. 7-9, 921-940.

[12] Z. Djadli, E. Hebey, and M. Ledoux, Paneitz-type operators and applications, Duke Mathematical Journal 104 (2000), no. 1, 129-169.

[13] Z. Djadli, A. Malchiodi, and M. O. Ahmedou, Prescribing a fourth order conformal invariant on the standard sphere. I. A perturbation result, Communications in Contemporary Mathematics 4 (2002), no. 3, 375-408.

[14] _ Prescribing a fourth order conformal invariant on the standard sphere. II. Blow up analysis and applications, Annali della Scuola Normale Superiore di Pisa. Classe di Scienze. Serie V 1 (2002), no. 2, 387-434. 
[15] F. Ebobisse and M. O. Ahmedou, On a nonlinear fourth-order elliptic equation involving the critical Sobolev exponent, Nonlinear Analysis. Theory, Methods \& Applications. An International Multidisciplinary Journal. Series A: Theory and Methods 52 (2003), no. 5, 1535-1552.

[16] D. E. Edmunds, D. Fortunato, and E. Jannelli, Critical exponents, critical dimensions and the biharmonic operator, Archive for Rational Mechanics and Analysis 112 (1990), no. 3, 269-289.

[17] V. Felli, Existence of conformal metrics on $S^{n}$ with prescribed fourth-order invariant, Advances in Differential Equations 7 (2002), no. 1, 47-76.

[18] J. García Azorero and I. Peral Alonso, On limits of solutions of elliptic problems with nearly critical exponent, Communications in Partial Differential Equations 17 (1992), no. 11-12, 2113-2126.

[19] F. Gazzola, H.-C. Grunau, and M. Squassina, Existence and nonexistence results for critical growth biharmonic elliptic equations, Calculus of Variations and Partial Differential Equations 18 (2003), no. 2, 117-143.

[20] D. Geng, On blow-up of positive solutions for a biharmonic equation involving nearly critical exponent, Communications in Partial Differential Equations 24 (1999), no. 11-12, 2333-2370.

[21] Z.-C. Han, Asymptotic approach to singular solutions for nonlinear elliptic equations involving critical Sobolev exponent, Annales de l'Institut Henri Poincaré. Analyse Non Linéaire 8 (1991), no. 2, 159-174.

[22] R. Lewandowski, Little holes and convergence of solutions of $-\Delta u=u^{(n+2) /(n-2)}$, Nonlinear Analysis. Theory, Methods \& Applications. An International Multidisciplinary Journal. Series A: Theory and Methods 14 (1990), no. 10, 873-888.

[23] C.-S. Lin, A classification of solutions of a conformally invariant fourth order equation in $\mathbf{R}^{n}$, Commentarii Mathematici Helvetici 73 (1998), no. 2, 206-231.

[24] O. Rey, Proof of two conjectures of H. Brézis and L. A. Peletier, Manuscripta Mathematica 65 (1989), no. 1, 19-37.

[25] Sobolev exponent, Journal of Functional Analysis 89 (1990), no. 1, 1-52.

[26] _ Blow-up points of solutions to elliptic equations with limiting nonlinearity, Differential and Integral Equations. An International Journal for Theory and Applications 4 (1991), no. 6, $1155-1167$.

[27] _ The topological impact of critical points at infinity in a variational problem with lack of compactness: the dimension 3, Advances in Differential Equations 4 (1999), no. 4, 581-616.

[28] R. C. A. M. Van der Vorst, Fourth-order elliptic equations with critical growth, Comptes Rendus de l'Académie des Sciences. Série I. Mathématique 320 (1995), no. 3, 295-299.

Khalil El Mehdi: Faculté des Sciences et Techniques, Université de Nouakchott, BP 5026,

Nouakchott, Mauritania

E-mail address: khalil@univ-nkc.mr

Current address: The Abdus Salam ICTP, 34014 Trieste, Italy 


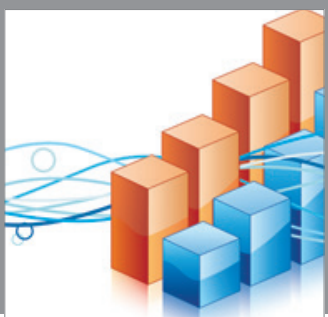

Advances in

Operations Research

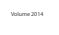

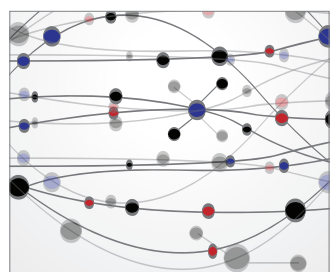

\section{The Scientific} World Journal
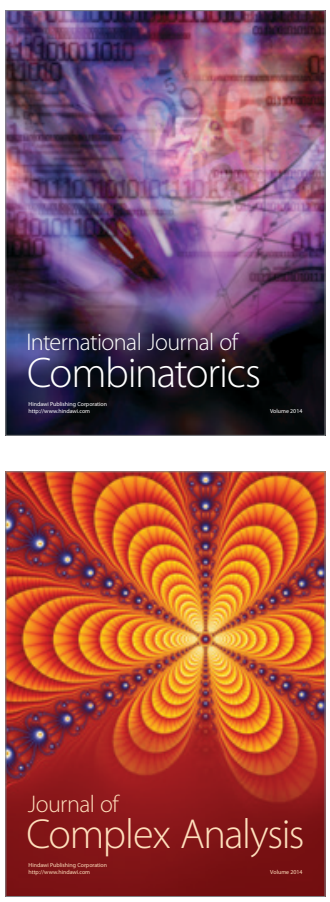

International Journal of

Mathematics and

Mathematical

Sciences
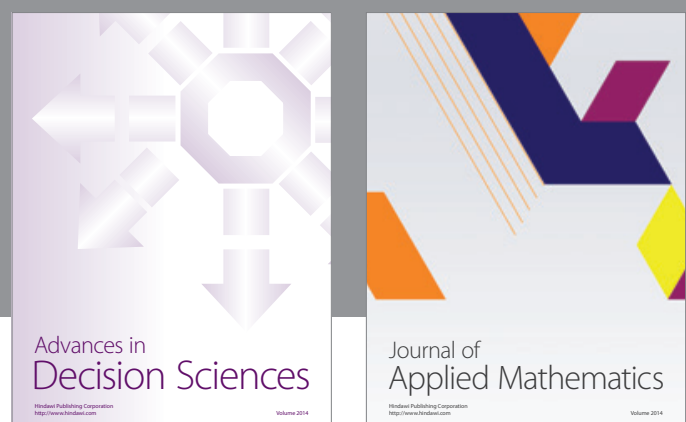

Journal of

Applied Mathematics
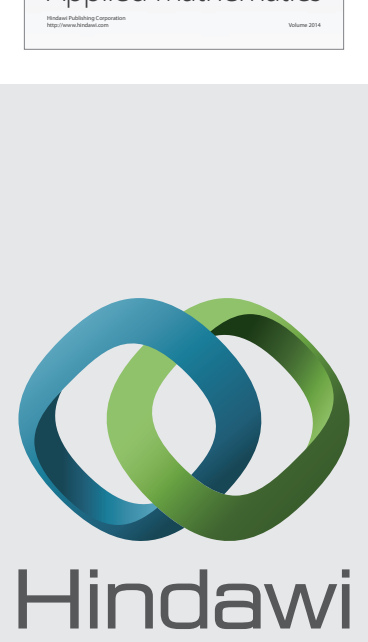

Submit your manuscripts at http://www.hindawi.com
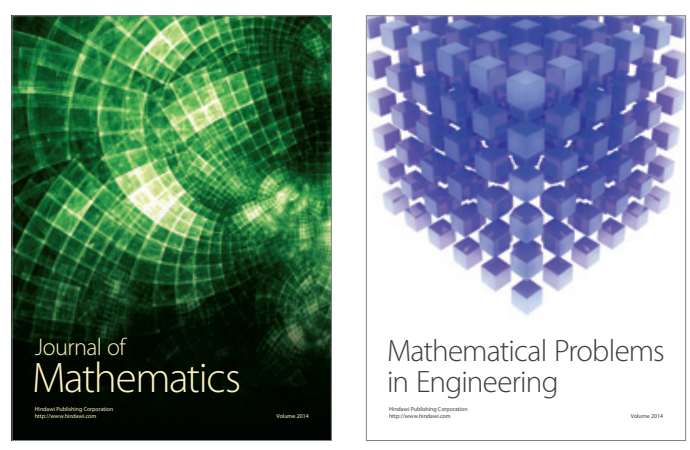

Mathematical Problems in Engineering
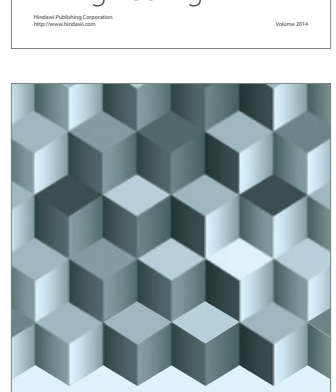

Journal of

Function Spaces
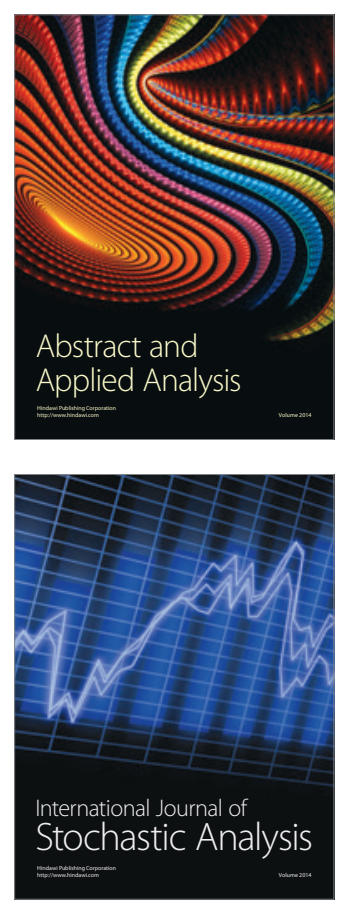

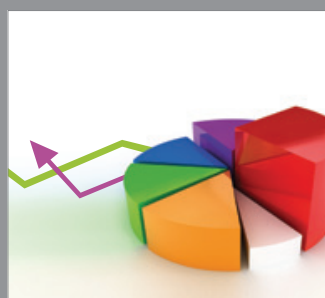

ournal of

Probability and Statistics

Promensencen
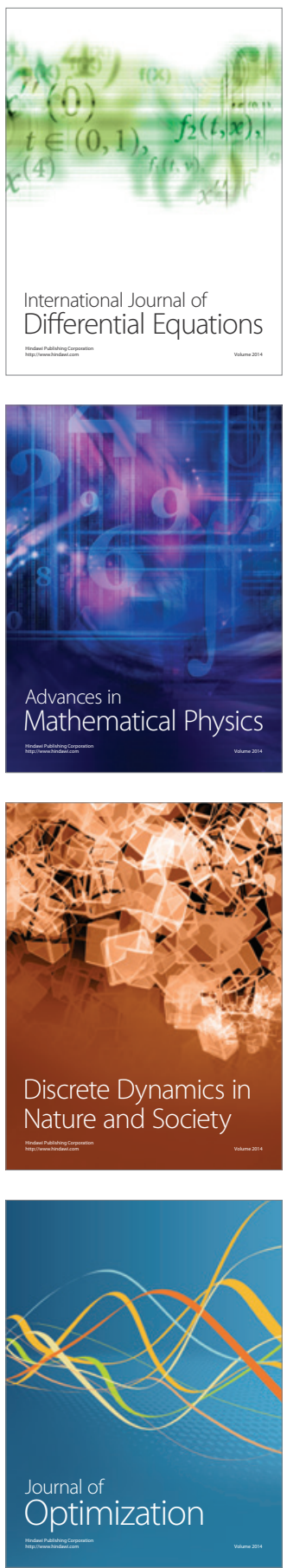